\title{
Fast Gating for Raman Spectroscopy
}

\author{
Andrea Chiuri (1) and Federico Angelini *
}

Diagnostic and Metrology Laboratory, FSN-TECFIS-DIM Nuclear Fusion and Safety Technologies Department, ENEA Via Enrico Fermi 45, 00044 Frascati, Italy; andrea.chiuri@enea.it

* Correspondence: federico.angelini@enea.it

\section{check for}

updates

Citation: Chiuri, A.; Angelini, F. Fast Gating for Raman Spectroscopy.

Sensors 2021, 21, 2579.

https://doi.org/10.3390/s21082579

Academic Editor: Clement Yuen

Received: 12 March 2021

Accepted: 2 April 2021

Published: 7 April 2021

Publisher's Note: MDPI stays neutral with regard to jurisdictional claims in published maps and institutional affiliations.

Copyright: (c) 2021 by the authors. Licensee MDPI, Basel, Switzerland. This article is an open access article distributed under the terms and conditions of the Creative Commons Attribution (CC BY) license (https:// creativecommons.org/licenses/by/ $4.0 /)$.

\begin{abstract}
Fast gating in Raman spectroscopy is used to reject the fluorescence contribution from the sample and/or the substrate. Several techniques have been set up in the last few decades aiming either to enhance the Raman signal (CARS, SERS or Resonant Raman scattering) or to cancel out the fluorescence contribution (SERDS), and a number of reviews have already been published on these sub-topics. However, for many reasons it is sometimes necessary to reject fluorescence in traditional Raman spectroscopy, and in the last few decades a variety of papers dealt with this issue, which is still challenging due to the time scales at stake (down to picoseconds). Fast gating ( $<1 \mathrm{~ns})$ in the time domain allows one to cut off part of the fluorescence signal and retrieve the best Raman signal, depending on the fluorescence lifetime of the sample and laser pulse duration. In particular, three different techniques have been developed to accomplish this task: optical Kerr cells, intensified Charge Coupling Devices and systems based on Single Photon Avalanche Photodiodes. The utility of time domain fast gating will be discussed, and In this work, the utility of time domain fast gating is discussed, as well as the performances of the mentioned techniques as reported in literature.
\end{abstract}

Keywords: time-gating; Raman; ICCD; Kerr; SPAD

\section{Introduction}

Raman spectroscopy is a powerful tool for chemical analysis, providing both sensitive and selective measurements [1]. On the other hand, a drawback of this technique is given by the generally small cross-section, and the possible overimposed fluorescence signal (both from the sample and the substrate) that possibly covers the weak Raman signal. As observed in [2], fluorescence occurs concurrently only with Stokes Raman lines, since anti-Stokes Raman lines are blue-shifted with respect to the excitation radiation. However, under normal conditions, only Stokes lines are visible, or in any case, they are the most intense and hence the most used for chemical identification.

To overcome this problem, a variety of techniques have been set over in the past few decades [3]. As summarized in that work, the possibilities range from suppressing fluorescence signal to enhancing the Raman signal, to other possibilities based on polarization or specific optical layouts. A graphic scheme of the classification is reported in Figure 1. Enhancement of Raman scattering can be obtained by coherent anti-Stokes Raman spectroscopy (CARS), surface enhanced Raman spectroscopy (SERS), Resonant Raman Scattering or stimulated Raman spectroscopy, while those aiming directly to eliminate the fluorescence contribution are shifted excitation Raman difference spectroscopy (SERDS), narrow spectral filtering, phase-sensitive detection and fast time gating. Extensive reviews on some of these subtopics can be found in [4-8]. However, no reviews have been published so far about the time-domain gating for Raman spectroscopy, a widely used technique for fluorescence suppression. In fact, most of the mentioned techniques require either sample preparation, or a specific wavelength selection for each compound under inspection. Moreover, sometimes further radiation is requested for the excitation of the energetic levels entering the Raman emission, causing photo-degradation of the sample. 

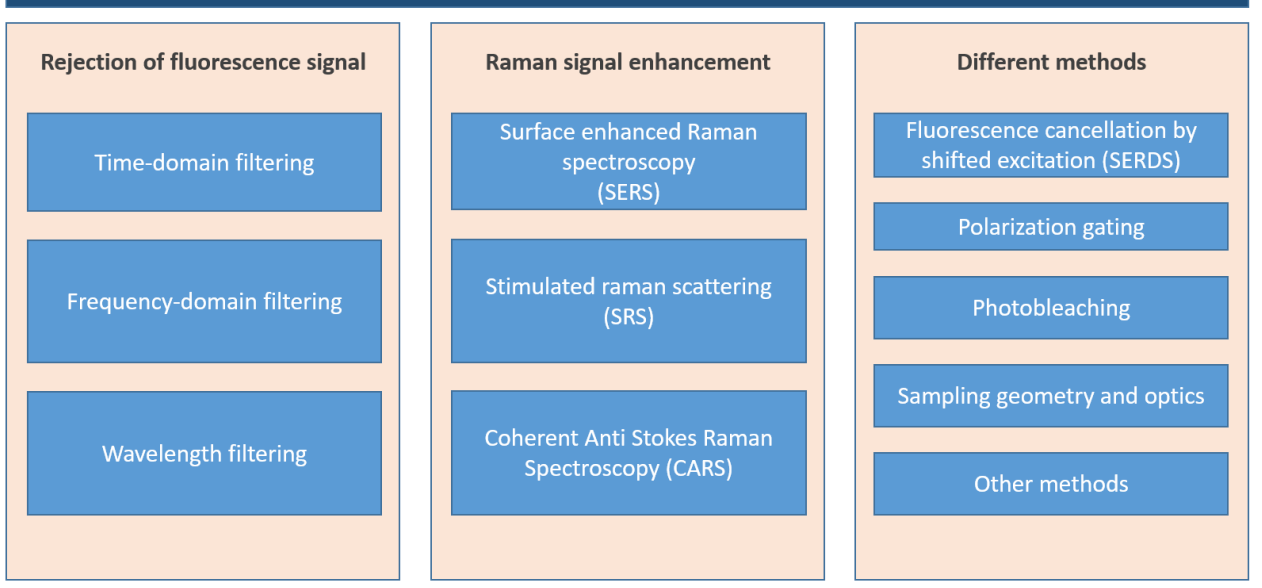

Figure 1. Graphic classification of methods for Raman enhancement regarding fluorescence signals.

The IEC 60825-1 international standard provides the guidelines to evaluate the maximum permitted exposition (MPE) to laser radiation according to a series of the beam parameters: energy, pulse duration, total exposure time, repetition rate, beam section, divergence and wavelength. Though a combination of single pulse energy and repetition rate can be found to maximize the Raman signal for a given exposure time in certain spectral regions [9], the problem of fluorescence is not bypassed, since fluorescence signal is increased as well, if UV radiation is employed. On the other hand, the IR window between 1500 and $1800 \mathrm{~nm}$ allows increasing the permitted dose, and in theory the Raman signal could be enhanced in a fluorescence-free scenario. However, the very small cross-section (100 times smaller than the $355 \mathrm{~nm}$ one), the performances of IR cameras and the low availability of lasers in this region make this choice currently hard to use, especially in biological samples where the strong water absorption could further weaken the Raman signal. Another important drawback is given by the very large Raman shift, in terms of wavelength, associated with Raman spectra in the IR, which obliges one to gather a very large spectral region, where optical efficiencies could be rather inhomogeneous: if $1540 \mathrm{~nm}$ excitation radiation is used, water vapor Stokes lines fall at about $3000 \mathrm{~nm}$.

Classic Raman spectroscopy represents then, in theory, the most convenient technique for the reliable identification of the largest number of species with a minimal exposition of the sample. As mentioned, the main drawback is the small cross-section, and fluorescence may become a problem, especially if short wavelengths $(250-400 \mathrm{~nm})$ are used to increase the Raman cross-section.

In theory, the spectral range below $270 \mathrm{~nm}$ can be used as a relatively fluorescence-free signal, since most fluorescence spectra start from about $270 \mathrm{~nm}$. However, in this region the absorption spectra may show sharp increases due to resonance, and no absorption below the resonance [10]. This causes nonlinearity in responses among different compounds, and limits the number of detectable species, since every compound may show resonance at a different wavelength. Hence, it cannot be suggested as a standard technique to overcome the fluorescence interference. Since the fluorescence spectra are usually much broader than Raman peaks, a solution could be to subtract the fluorescence as a baseline, provided that the signal is also collected at other wavelengths close to the Raman one. On the other hand, a narrow spectral filtering allows one to eliminate most of the fluorescence signal, which is emitted at different wavelengths. Nevertheless, because of the Poisson process of light measurement, the signal variance equals its average value: this means that the fluctuations associated with large signals may mask weaker signals overimposed on them.

Fortunately, other methods allow one to avoid fluorescence, as mentioned before. Another method employs temporal gating to exploit the slower timescales of fluorescence with respect to the Raman emission. Three techniques are currently used for fast gating. 
The first is the use of a time-gated intensified CCD (ICCD), where the signal emitted from a photocatode is amplified only inside a short time interval and includes the light pulse (in general from a laser). This method has been proven as effective in rejecting fluorescence [11]. Nowadays, gating times as short as $200 \mathrm{ps}$ are available on the market, so that a great part of the fluorescence echo can be cut off. However, 200 ps does not provide excellent fluorescence rejection for fluorophores with short decay times. In any case, ICCDs are very expensive instruments, and exclusively coupled to a CCD, which is not necessary the best detector in any application.

To reduce the cost and improve the integration capability of the detector, time-gated single photon avalanche diode (TG-SPAD)-based CMOS pixel circuits are a possible solution. These very fast detectors are able to detect single photons and allow gating times as short as 100 ps and less.

The third method employs an optical shutter (e.g., the Kerr cell, the Pockels cell or a photoacusitc cell) to allow light transmission only in a short time lapse. Among all these effect, a sharp change in the optical properties of a medium (crystalline or even amorphous) causes the birefringence of the medium which, if appropriately exploited, lets the medium become transmissive for a short time. The Kerr effect, in particular, can be driven by the laser light itself (optical Kerr effect) so that a single laser pulse may be used to excite the Raman spectrum and gate the detection, obtaining time gates as fast as the laser pulses. If a picosecond laser is employed, picosecond gating times can be easily reached. Nevertheless, this technique has the disadvantage of single-channel detection, which requires a scan of wavelengths to acquire a complete spectrum. In the following the advantages and drawbacks of each method will be discussed. In Section 2, some theoretical considerations about the performances achievable with fast-gated Raman detectors are presented. In Section 3, an overview of the gate based upon the optical Kerr cell is given, and theoretical calculations about the efficiency of this approach are discussed. Sections 4 and 5 describe the setups employing single photon avalanche photodiodes and intensified CCDs, even coupled to a streak camera. A summary of the contents, and final remarks about the performances, pros and cons of each technique, is finally given in Section 6.

\section{Fast Gating: Expected Performances}

A simple model can be set up to establish which part of a fluorescence signal can be cut off by a given time gate. The scheme of fluorescence emission is described through the Jablonsky diagrams. While the Raman signal can be assumed as instantaneous, fluorescence build-up time is typically of the order of picoseconds [2], as shown in Figure 2. During this period, called the internal conversion period, a series of non-radiative processes leads the electronic states to the lowest excited electronic level, and then the fluorescence consists of the return to the ground electronic state (although excited vibrational levels are possible), and the result is a series of photons of different energies, or under a macroscopic point of view, light of different wavelengths because of the many different transitions between excited vibrational states of the upper electronic level and the excited vibrational levels of the ground state.

This process of re-emission happens in times spanning from less than a nanosecond to hundreds of nanoseconds (the fluorescence lifetime $\tau_{f}$ ), and exponential decay is generally observed for fluorescence intensity for each fluorophore. Unfortunately, the fluorescence signal may overpass the Raman one by several orders of magnitude, and its intrinsic fluctuations significantly lower the signal to noise ratio achievable in Raman detection.

When a laser pulse hits a fluorophore, the convolution between the laser intensity and the fluorescence decay determines the temporal shape of the signal re-emitted by the sample. In Figure 3, the total Raman (blue) and the fluorescence (red) signals are plotted versus time together with the total signal (yellow), for $0.1 \mathrm{~ns}$ full width half maximum (FWHM) pulse width. The laser pulse intensity can be generally described as a double exponential $[12,13]$, but by following [14] it can be approximated by a triangular function 
of time, as shown in Figure 4, for better control of the effects of function parameters on the signal properties (area, symmetry, duration).

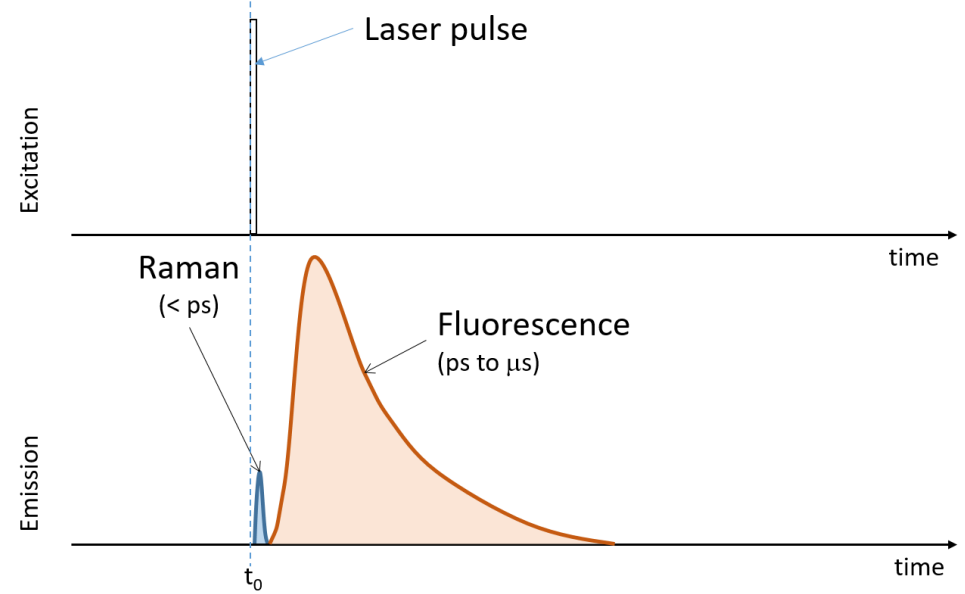

Figure 2. Temporal profile of the excitation laser pulse, emitted Raman scattering signal and emitted fluorescence.
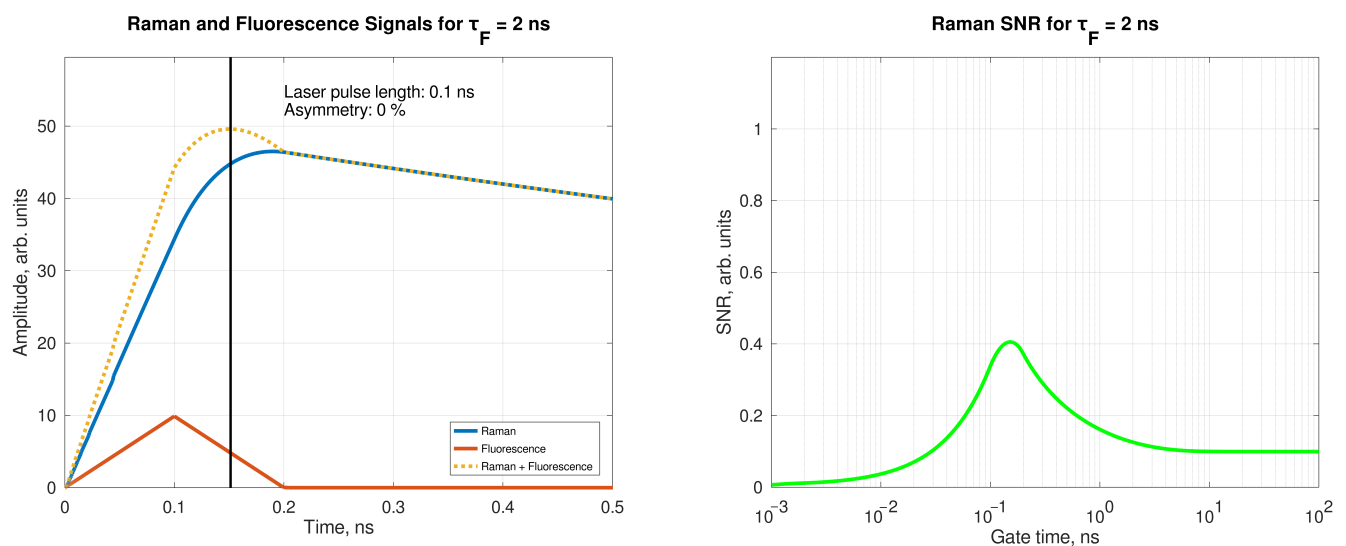

Figure 3. The Raman, fluorescence and total (Raman + fluorescence) signals as a function of time (left) and the Raman signal-to-noise ratio (RSN) as a function of the gating time. The black line in the left plot represents the gating time at which the best RSN is obtained, i.e., the abscissa of the maximum in the (right) plot.

In this study the fluorescence signal is imposed to be 100 times stronger than the Raman one; this latter is obtained as the difference between the total signal and the fluorescence signal (the two measurable quantities), and a proxy of the performances of the rejection system can be calculated as the Raman $/ \sqrt{\text { Total }}$. This quantity can be defined as Raman signal-to-noise ratio (RSN)m and it depends on the gating time. In the best case, Raman is completely detected and fluorescence is completely rejected; this value will equal 1. In the worst case where all the Raman and fluorescence signals are detected it will equal $1 / \sqrt{101}=0.0995$.

In fact, if the signal is integrated over a very short time $(<<$ FWHM), neither Raman nor fluorescence signals will be detected, giving a very small RSN. On the other hand, if the gating time is very long $(\approx \mu \mathrm{s})$, all the fluorescence signal will be detected, and the error associated with the measurement will be ten times the Raman signal itself. Between these two extreme choices, a gating time close to the laser pulse length may be imposed to stop the acquisition, and the Raman signal will become more visible, according to the laser pulse length and the fluorescence lifetime. It is intuitive that the longer the fluorescence decay, the easiest the fluorescence cutoff. However, a complete rejection of fluorescence is not possible, unless the laser pulses are shorter than $\approx$ ps; in this case, however, the spectral 
broadening associated with short pulses makes the spectral resolution too low for effective Raman detection.

Additionally, the shape of the laser pulse could influence the ability of rejection: If the peak is very close to the beginning (a realistic behavior) the rejection ability is expected to be better than for a symmetric pulse. An asymmetry factor $\approx$ may be defined as the ratio between the distance between the beginning of the signal and the peak and the middle point of the signal. In terms of the points defined in Figure $4, \alpha=\overline{C B} / \overline{A C}$.

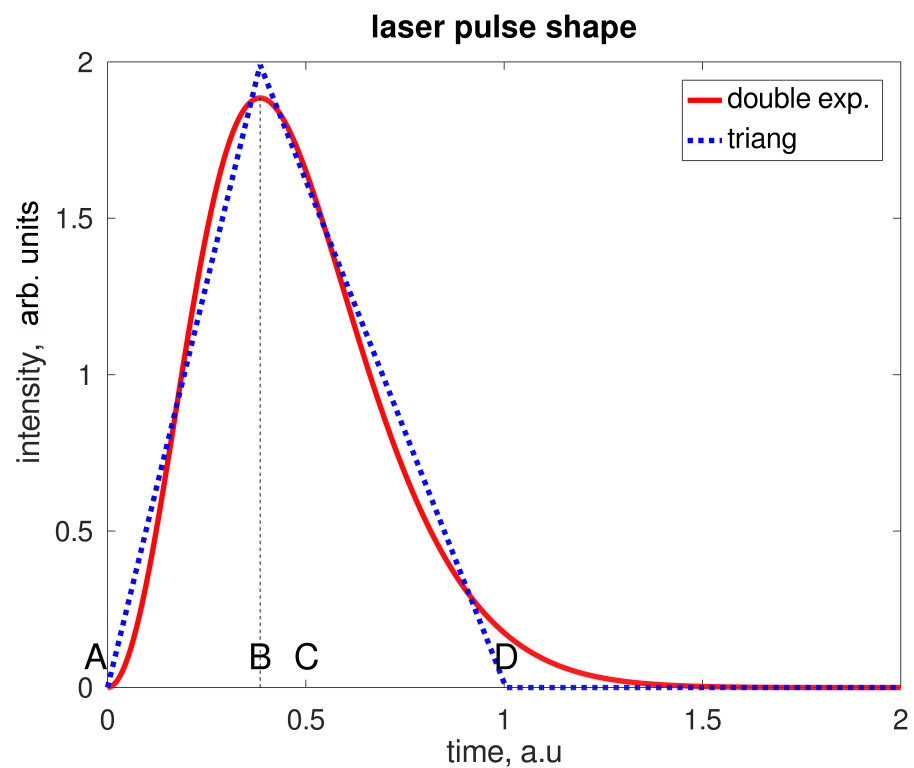

Figure 4. Approximation of a double exponential laser pulse with a triangular function having an equal area. The parameters of the triangular function are determined so that the modal value is the same as that of the double exponential, and to ensure an even ratio for the areas to the left and to the right of the modal value (indicated by the dotted line). Point A represents the beginning of the pulse; $B$ is the time where the peak value is reached; $C$ is the middle point between A and D; and D is the end point of the triangular pulse.

A symmetric pulse has a null asymmetry factor. However, the effects of the pulse shape on the performances reached by the time-gated spectroscopy are not so significant. The model calculates the best RSN according to laser pulse length FWHM, fluorescence lifetime $\tau_{f}$ and the asymmetry factor of the laser pulse. As a first result, it can be inferred that the best RSN is always achieved for gating times about 1.7 times the laser FWHM, with only a marginal impact due to the asymmetry of the pulse. Only shapes where B is found to the left of $\mathrm{C}$ are considered; results are shown in Table 1.

Table 1. Coefficients between pulse length full width half maximum (FWHM) and best gating time for some values of a. The values represent the angular coefficients of the linear regression between the best gating time and the laser pulse width FWHM. To evaluate the goodness of linear fit, the $r^{2}$ coefficients are also shown.

\begin{tabular}{cccccc}
\hline Asymmetry Factor $\boldsymbol{\alpha}$ & $\mathbf{0}$ & $\mathbf{0 . 2}$ & $\mathbf{0 . 4}$ & $\mathbf{0 . 6}$ & $\mathbf{0 . 8}$ \\
\hline Best gating/FWHM & 1.72 & 1.68 & 1.66 & 1.65 & 1.65 \\
$r^{2}$ & 0.99922 & 0.99881 & 0.99801 & 0.99734 & 0.99723 \\
\hline
\end{tabular}

Another result is shown in Figure 5, where the best RSN achievable is plotted against the fluorescence lifetime and the FWHM. It is evident that laser pulses longer than $100 \mathrm{ps}$ never allow a complete fluorescence cutoff, especially for short-lived fluorophores.

From these results the advantages of an optical Kerr cell are evident, as the gating time can be kept as short as the laser pulse: employing picosecond lasers this would 
enable very high RSNs. Some details on the proposed methods will be described in the following sections.

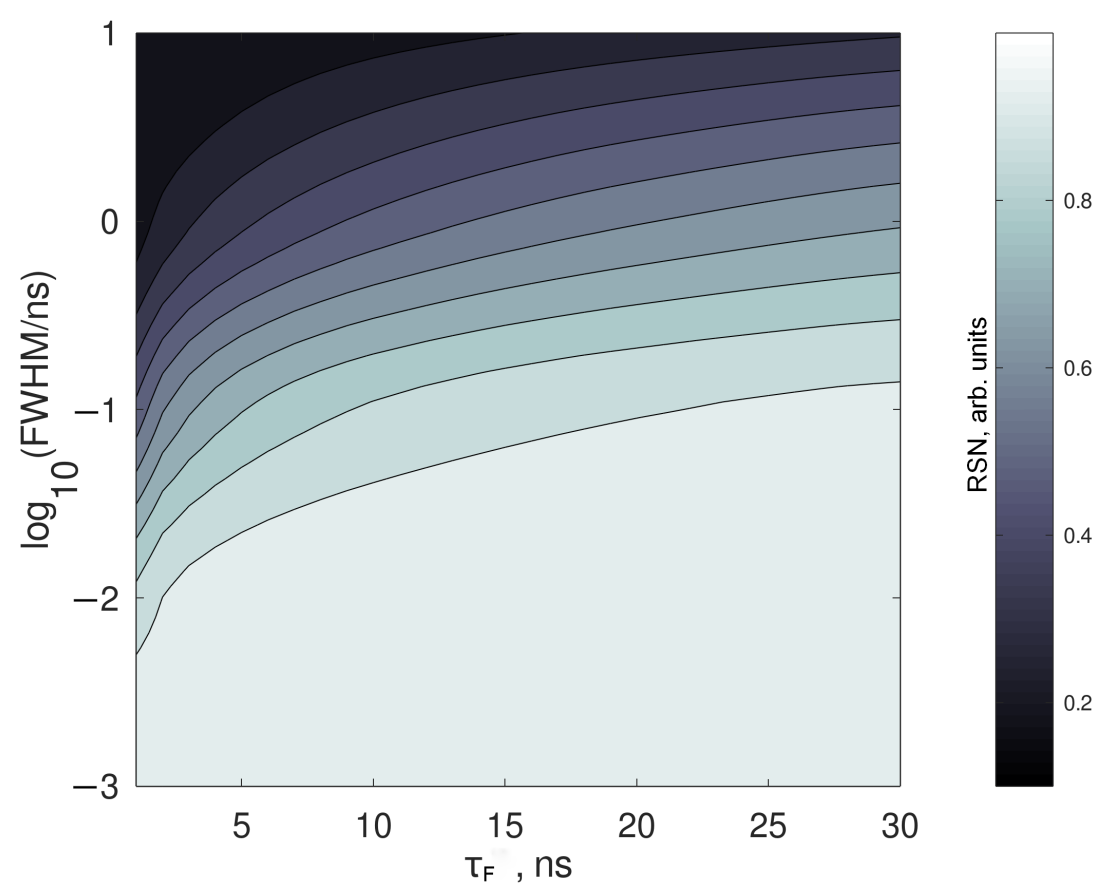

Figure 5. The best RSN achievable in a time-gated Raman system when a 100-times stronger fluorescence signal is overimposed to Raman signal, as a function of the fluorescence decay time and the laser full width half maximum pulse length. The gating time is always about $1.7 \times$ FWHM. Pulse asymmetries do not significantly change the results.

\section{Optical Kerr Gate}

Some materials are subject to changes in optical properties when subjected to an electric field $[15,16]$. In fact, electromagnetic forces may alter the microphysical properties of atoms and molecules of the material (position, orientation or shape). This is reflected in changes in the refractive index, for example, after the application of a low-frequency electric field. If the material is anisotropic, the field applied modifies its refractive indices and its behavior in polarized light.

The dependence of the refractive index on the applied electric field takes one of two forms:

- The refractive index changes in proportion to the applied electric field, in which case the effect is known as the linear electro-optic effect or the Pockels effect. The linear electro-optical effect can be described in terms of a nonlinear polarization given by:

$$
P_{i}(\omega)=2 \sum_{j k} \chi_{i j k}^{(2)}(\omega+0) E_{j}(\omega) E_{k}(0)
$$

since the linear electro-optic effect can be described by a second-order nonlinear susceptibility [15], it follows that a linear electro-optic effect can occur only for materials that are non-centrosymmetric (i.e., they do not display inversion symmetry). In fact, for centrosymmetric ones $\chi^{(2)}=0$ and $\chi^{(3)} \neq 0$; for non-centrosymmetric, $\chi^{(2)} \neq 0$ and $\chi^{(3)} \neq 0$ (with $c h i^{(2)} \operatorname{chi}^{(3)}$ ). Furthermore, in this case a refractive index ellipsoid should be defined; hence, the material cannot be amorphous (i.e., with the same refractive index along each direction) and only crystals could be considered. Since in this case there is only a linear variation of the refractive index, the centrosymmetric crystals do not display this effect because of their inversion symmetry. Nowadays, very fast Pockels cells are produced by some manufacturers, who declare gating 
times as short as $150 \mathrm{ps}$, with window diameters up to $6 \mathrm{~mm}$. These devices could represent a promising tool for fast Raman gating, though no research articles have been published so far on this topic.

- The refractive index changes in proportion to the square of the applied electric field, in which case the effect is known as the quadratic electro-optic effect or the Kerr effect. This occurs in centrosymmetric materials (i.e., they display inversion symmetry), where the lowest-order change in the refractive index depends quadratically on the strength of the applied field. It can be described in terms of a nonlinear polarization given by:

$$
P_{i}(\omega)=3 \sum_{j k l} \chi_{i j k l}^{(3)}(\omega+0+0) E_{j}(\omega) E_{k}(0) E_{l}(0)
$$

Due to dependence by $\chi^{(3)}$, the non-linearity can be induced by placing the medium inside DC or AC electric fields (e.g., a sort of capacitor can be used); otherwise, the electric field can be generated by a strong radiation (e.g., a laser beam). The latter, usually known as the optical Kerr effect, allows one to improve the performances, and lower gating windows (e.g., ps) could be achieved.

In any case, refractive index variations induced in this way are usually very small. Nevertheless, they can induce significant effects if the wave propagates over distances much larger than its wavelength, so that small phase changes may induce measurable total phase shifts. As an example, for a refractive index change of $10^{-5}$, an optical wave will experience an additional shift of $2 \pi$ after $10^{5}$ wavelengths. These materials can be used to realize electrically controlled optical devices-for example:

- Lenses, where the the focal length can be varied changing the refractive index;

- Prisms, where the beam deviation is controllable by changing the refractive index, becoming devices capable of scanning;

- Phase modulators, where light transmitted undergoes a controllable phase shift;

- Wave retarders, where anisotropic crystals may become able to change the polarization properties of the beam crossing the medium;

- Optical switches, where wave retarders between two crossed polarizers cause transmission of the whole device to be dependent on the phase shift introduced.

Based on the idea expressed with the last example, the Kerr effect was employed for time-resolved Raman spectroscopy (TRRS) [3,17-21].

One of the first experiments [17] was based on a Kerr gate with a resolution of few picoseconds at a $102 \mathrm{~Hz}$ repetition rate and allowed the experimenters to detect light with a spectrometer and CCD combination. These systems achieved a good suppression of the background from fluorophore with a lifetime of about 2 ns.

As previously described, in a Kerr cell the non-linearity of an optical medium is exploited to introduce an optical phase delay and to rotate the polarization of the impinging beam. In this work the non-linearity induced by a laser beam will be considered since this approach paves the way for the use of the same laser pulse to stimulate the Raman signal and to activate the cell (i.e., optical Kerr effect). In this way, the fluorescence, which occurs after a few picoseconds, can be rejected with very high efficiency at the end of the laser pulse.

Furthermore, the optical pumping allows one to use any pulse repetition rate (PRR) to integrate the signal, since no electronic trigger is required during the acquisition phase. On the other hand, the use of very high PRR and very short pulse lasers (up to picoseconds) should be preferred, in order to increase the signal since the single pulse energy is usually not very high.

\subsection{Nonlinear Refractive Index}

The Kerr effect relies on the non-linearity induced into a medium. Let us introduce the refractive index as $n=n_{0}+\Delta n$, where $n_{0}$ is the linear term, while $\Delta n=n_{2} \cdot E^{2}$ is a function of the incident field $\mathrm{E}$ (i.e., a laser) and the nonlinear refractive index $n_{2}$ [15]. By 
exploiting these properties, it is possible to introduce a phase $\phi$ between two orthogonal components of a polarized beam which can be expressed as follows:

$$
\phi=\frac{2 \pi \Delta n}{\lambda} L=\frac{2 \pi n_{2}}{\lambda} L I
$$

where $L$ : thickness of the nonlinear medium; $\lambda$ : wavelength of the gated pulse- that is, the Raman signal to be rotated by the Kerr medium, as will be described in the next paragraphs; I: power density of the pulse used to activate the gate (i.e., the nonlinearity in the crystal). Let us introduce the definition of the power density $I=\frac{E}{T \pi\left(\frac{d}{2}\right)^{2}}$, where $E$ : pulse energy; $T$ : pulse duration; $d$ : laser beam diameter within the nonlinear medium. This leads to a more useful expression for the phase $\phi$ :

$$
\phi=\frac{8 L E n_{2}}{\lambda T d^{2}}
$$

By suitably varying the parameters reported in Equation (4) it is possible to create a half-wave plate (i.e., $\phi=\pi$ ). In this case, if the crystal is rotated by an angle $\theta$, the polarization of the emerging beam will be rotated by an angle by $2 \cdot \theta$. It is worth noting that the active medium will act as an half-wave plate only for one value of $\lambda$, once given the other parameters. For this reason, in Section 3.3 we will define a transfer function characterizing the Kerr cell efficiency at different wavelengths. Since the parameters included in Equation (4) represent physical properties with intrinsic uncertainty, they cause a variety of phase $\phi$; hence, they will be considered in order to characterize the efficiency of a real setup.

\subsection{Experimental Implementation}

Let us now introduce the experimental scheme reported in Figure 6 which could be exploited to generate an optical Kerr gate devoted to the TRRS.

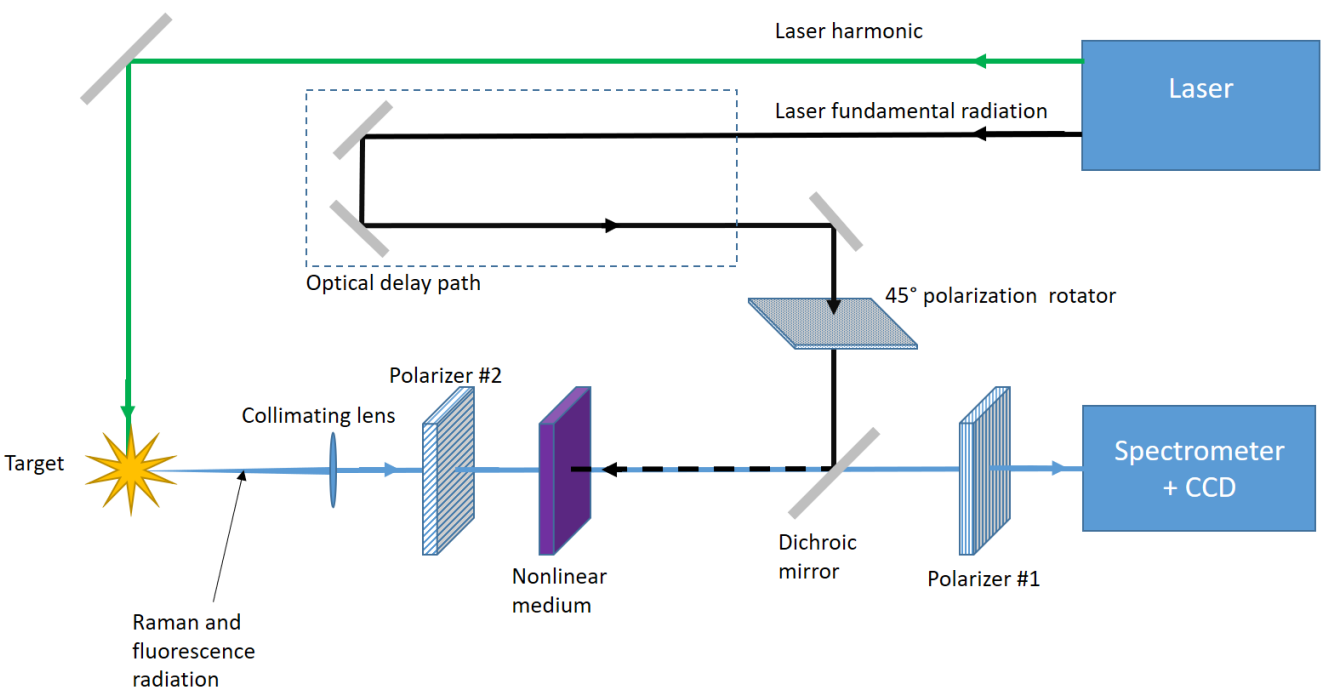

Figure 6. Experimental setup of a Kerr cell optically activated exploiting the properties of a nonlinear crystal.

The fundamental optical elements are [15,16]:

- $\quad$ The polarizer \#1 (e.g., a polarizing beam splitter-PBS).

- $\quad$ The polarizer \#2.

- The nonlinear medium.

- A laser able to emit at two different wavelengths, the first to excite the target (i.e., Raman Signal) and the second to induce the Kerr effect. 
The Raman signal (blue in Figure 6) and the fluorescence impinge on the first polarizer and the emerging field oscillates along a well-defined plane. The pumping pulse (black in Figure 6), which induces the necessary birefringence within the active medium (i.e., the crystal), should be suitably synchronized with the Raman radiation. This is usually accomplished by a simple optical variable delay line obtained with mobile mirrors. Since the Kerr effect relies on high energy density, the active medium is usually pumped by exploiting the laser fundamental harmonic, while lower wavelengths are necessary to obtain a higher Raman signal.

The active medium should act as a half-wave plate (i.e., $\lambda / 2$ ) at $45^{\circ}$ only during the pulse duration of the pumping laser; hence, the polarization of the radiation passing through the crystal in this time interval is rotated by $90^{\circ}$. The second polarizer, orthogonal with respect to the first one, allows one to select the Raman signal which was suitably rotated and to discard the undesired fluorescence.

In order to obtain a half-wave plate at $45^{\circ}$, you could rotate the crystal or you can follow a different approach, reported in Figure 6, where the gating pulse passes through a polarizer at $45^{\circ}$ or is rotated by a half-wave plate at $45^{\circ}$. In this case the birefringence generated in the nonlinear crystal allows one to realize a result equivalent to that achievable by rotating the active medium.

Several schemes of optical Kerr gate devoted to the TRRS were reported in literature [3,17-20]. The choice of a particular setup could be based on reasons related to the cost, technical characteristics (i.e., weight, dimensions, power) or just the availability on the market. More precisely:

- Most setups involve a picosecond laser, such as diodes @ 1064 nm [19] or solid state systems at $800 \mathrm{~nm}$ (e.g., Ti:sapphire laser) [17,18,22].

- Even though the same laser is usually exploited to obtain the Kerr effect and for the Raman signal, for reasons of costs and synchronization (in this case, doubledfrequency radiation should be generated), there is not a theoretical constraint on the use of different wavelength combinations. However, further assessments should be considered in order to get as many Raman signals as possible. A low absorbance of the active medium in the chosen range, and a Raman pump close to the UV to increase the cross-section would be suitable. A solution commercially available could involve a gating pulse at $1064 \mathrm{~nm}$ and a Raman pump at $532 \mathrm{~nm}$, i.e., a gated pulse close to $532 \mathrm{~nm}$ or both the gating and gated pulses at $532 \mathrm{~nm}$. In the present paper we will focus on this easier configuration.

- $\quad$ The active medium could be a liquid $\left(\mathrm{CS}_{2}\right)[17,18,23,24]$ or a solid $\left(\mathrm{TiO}_{2}, \mathrm{CdS}, \mathrm{ZnO}\right.$, bismuth glass) $[19,20]$. Several devices were optimized in order to exploit the $\mathrm{TiO}_{2}$ by varying the size and the configuration of the active medium, which could be homogenous or variously dispersed. Nevertheless, the choice of the active medium is based on the value of the nonlinear refractive index which should be as high as possible.

In this work, some simulations have been performed considering $\mathrm{TiO}_{2}$. This could be a good candidate for a setup devoted to the TRRS, since it has low absorbance in the visible range [25-30], which increases in the UV range, and it is less affected by problems than other media, e.g., low two-photon absorbance, because of the higher energy gap. In fact, multi-photon absorption of the gate pulse in the Kerr medium can cause a strong background emission [31].

\subsection{Kerr Gate Efficiency}

In this section, we report a performance analysis of the setup from Figure 6 by considering not only ideal configurations but even real conditions achievable with commercial tools and optical elements (e.g., laser, active medium and PBS).

The phase factor reported in Equation (4) should be as close as possible to $\pi$ in order to obtain an optimal gate. This condition can be achieved only for one $\lambda$ by suitably adapting the involved parameters which are characterized by intrinsic uncertainty. 
The total efficiency of the Kerr gate can be estimated by considering the Jones matrix formalism [32] for nonlinear optical elements $(L R(\phi))$ introducing a general phase factor $\phi$ between two orthogonal polarizations (i.e., horizontal and vertical), which is rotated by angles $\theta(R(\theta)$ and $R(-\theta))$ :

$L R(\phi, \theta)=R(-\theta) L R(\phi) R(\theta)=\left(\begin{array}{cc}\cos (-\theta) & \sin (-\theta) \\ -\sin (-\theta) & \cos (-\theta)\end{array}\right) \cdot\left(\begin{array}{cc}1 & 0 \\ 0 & e^{-i \phi}\end{array}\right) \cdot\left(\begin{array}{cc}\cos (\theta) & \sin (\theta) \\ -\sin (\theta) & \cos (\theta)\end{array}\right)$

Let us consider the usual orthogonal base for the polarization state composed by the vector $\left(\begin{array}{l}1 \\ 0\end{array}\right)$ for the horizontal state and $\left(\begin{array}{l}0 \\ 1\end{array}\right)$ for the vertical one. The PBS\#1 selects only one polarization state; here the state $\left(\begin{array}{l}1 \\ 0\end{array}\right)$ is considered but this model is valid even with a different choice. Hence, the output of the system composed by PBS\#1 and active medium is given by the following product:

$$
\left(\begin{array}{l}
\text { out }_{H} \\
\text { out }_{V}
\end{array}\right)=\operatorname{LR}(\phi, \theta)\left(\begin{array}{l}
1 \\
0
\end{array}\right)
$$

and $\left|o u t_{V}\right|$ is related to the the probability to have an output field vertically polarized $\left(\operatorname{prob}_{V}\right)$, i.e., the only state which can pass the PBS\#2.

It follows that the total efficiency can be given as the product:

$$
\eta_{t o t}=\operatorname{prob}_{V} \cdot \eta_{P B S} \cdot \eta_{A M} \cdot \eta_{\text {rot }}
$$

where $\eta_{A M}$ depends on the transmittance of the active medium (e.g., $\eta_{A M}=0.85$ for the $\mathrm{TiO}_{2}$ at $\left.532 \mathrm{~nm}[29,30]\right), \eta_{P B S}$ quantifies the efficiency of the PBS\#2 (commercial PBS achieves an extinction ratio of 1:1000; hence $\left.\eta_{P B S}=0.999\right)$ and $\eta_{\text {rot }}$ represents the efficiency of the rotation at $45^{\circ}$ of the active medium or the errors which can be made in equivalent experimental configurations.

This simple model can be used to estimate the efficiency for the whole Raman signal passing through the crystal, since the peak is narrow and the same properties (e.g., the transmittance of the active medium, the transmittance of the PBS, $n_{2}$ and $n$ are roughly constant) can be considered for all the wavelengths.

\subsection{Setup Design}

Let us consider the results obtained in Equation (7) and the values for the parameters reported in Equation (2) based on those of commercial lasers. The other terms are: $d=1 \mathrm{~mm}$ (not focused beam), $L=1 \mathrm{~mm}, \lambda=532 \mathrm{~nm}$ and $\mathrm{TiO}_{2}$ as active medium. In this case a low efficiency (i.e., $<0.001 \%$ ) is achieved even for short $\mathrm{T}(1 \mathrm{ps})$ and high $\mathrm{E}(500 \mathrm{~nJ})$ (see Figure 7a). Better performances can be obtained by varying $d$ within the diffraction limit (see Figure 7b), even though, in this case, it should be difficult to guarantee optimal overlap between the gating and the gated pulses. Different active media or other configurations of $\mathrm{TiO}_{2}$ [17-20,23-30] would bring about results similar to those reported in this section.

We can conclude that $d=10 \mu \mathrm{m}$ is a feasible value, although commercial beam shapers could guarantee a lower size, up to $1 \mu \mathrm{m}$. The other relevant parameter, E, should be as high as possible and depends on the peak power of the particular system. Nevertheless, high average and peak power of laser radiation (e.g., MW) cannot be considered, because that can induce sample damage and nonlinear processes. Figure 8 demonstrates that by varying only E from 1 to $100 \mathrm{~nJ}$, the same setup could reach the maximum achievable efficiency.

By exploiting Equation (4), it is possible to estimate the pulse energy needed to obtain a phase $\phi=\pi$ as $E=\pi d^{2} \lambda T / 8 L n_{2}$. In Table 2 we report several values of $E$ for different configurations (i.e., pulse diameter and pulse duration) of a Kerr cell based on $\mathrm{TiO}_{2}$ as active medium. 

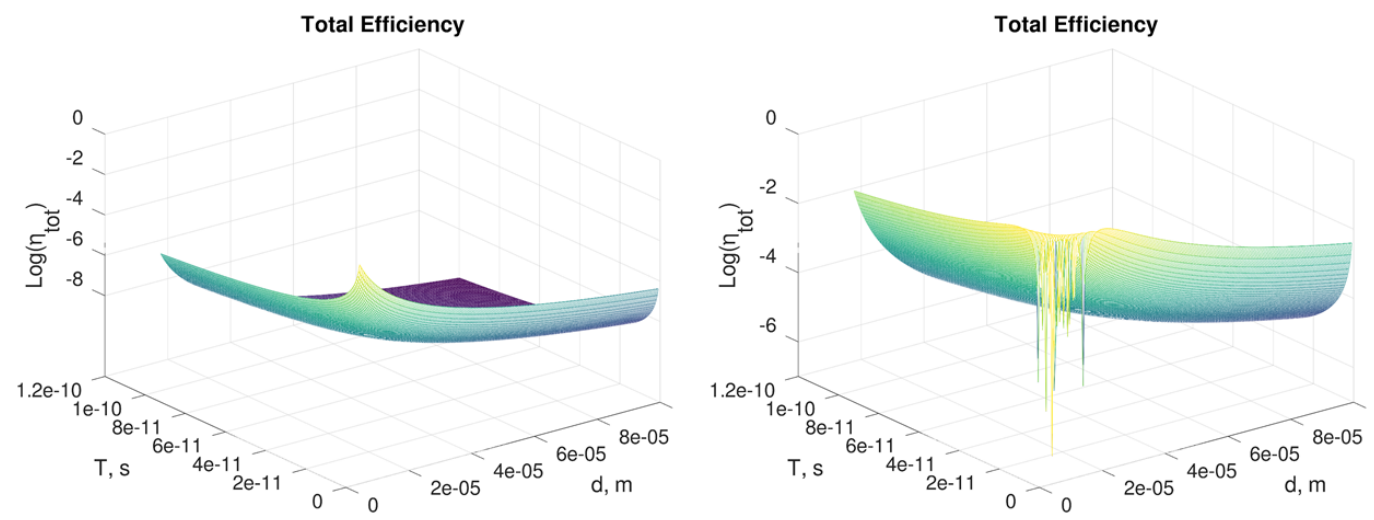

Figure 7. Left: $\log \left(\eta_{\text {tot }}\right)$ vs. E,T; right: $\log \left(\eta_{\text {tot }}\right)$ vs. E,d.
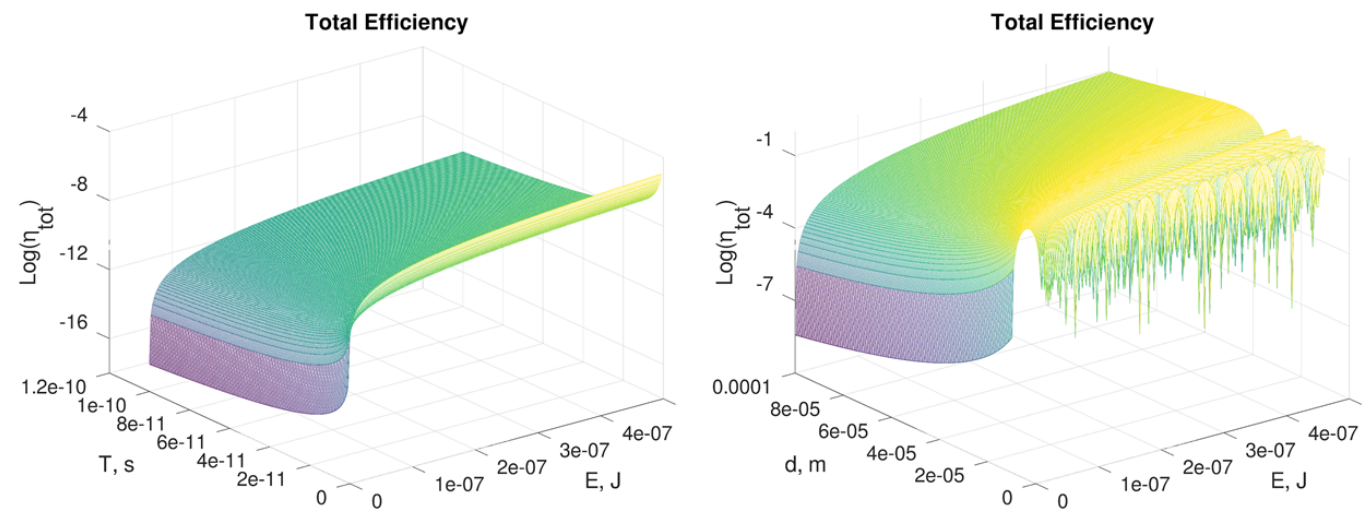

Figure 8. Left: $\log \left(\eta_{t o t}\right)$ vs. T,d $(\mathrm{E}=1 \mathrm{~nJ})$; right: $\log \left(\eta_{t o t}\right)$ vs. $\mathrm{T}, \mathrm{d}(\mathrm{E}=100 \mathrm{~nJ})$.

Table 2. Pulse energy (J) to obtain $\phi=\pi$ in a Kerr cell (active medium: $1 \mathrm{~mm}$ of $\mathrm{TiO}_{2}$ ) at $532 \mathrm{~nm}$.

\begin{tabular}{|c|c|c|c|c|}
\hline $\mathrm{T}[\mathrm{ps}] \quad \mathrm{d}[\mu \mathrm{m}]$ & 1 & 10 & 100 & 1000 \\
\hline 1 & $2.358 \times 10^{-10}$ & $2.358 \times 10^{-8}$ & $2.358 \times 10^{-6}$ & $2.358 \times 10^{-4}$ \\
\hline 10 & $2.358 \times 10^{-9}$ & $2.358 \times 10^{-7}$ & $2.358 \times 10^{-5}$ & $2.358 \times 10^{-3}$ \\
\hline 100 & $2.358 \times 10^{-8}$ & $2.358 \times 10^{-6}$ & $2.358 \times 10^{-4}$ & $2.358 \times 10^{-2}$ \\
\hline
\end{tabular}

Several kinds of commercial lasers are available in the range $T=[1,100]$ ps characterized by technical specifications suitable for a TRRS. It is worth noting that, in these cases, $d$ should be very low. A few examples, without citing specific laser manufacturers or suppliers, follow.

- $\quad \mathrm{PRR}=100 \mathrm{MHz}, \mathrm{T} \approx 45 \mathrm{ps}, \mathrm{W}>0.1 \mathrm{~W}, \mathrm{E}>1 \mathrm{~nJ}-\eta_{\text {tot }}=1.8 \%$ with $\mathrm{d}=1 \mu \mathrm{m}$ and $<<1 \%$ with $\mathrm{d}=10 \mu \mathrm{m}$.

- $\quad \mathrm{PRR}=40 \mathrm{MHz}, \mathrm{T}<9 \mathrm{ps}, \mathrm{W}>0.06 \mathrm{~W}, \mathrm{E}>1.5 \mathrm{~nJ}-\eta_{\text {tot }}=68.2 \%$ with $\mathrm{d}=1 \mu \mathrm{m}$ and $0.01 \%$ with $\mathrm{d}=10 \mu \mathrm{m}$.

- $\quad \mathrm{PRR}=1-80 \mathrm{MHz}, \mathrm{T}=88-96 \mathrm{ps}, \mathrm{W}=0.0063-0.34 \mathrm{~W}, \mathrm{E}=4.3-6.3 \mathrm{~nJ}-\eta_{\text {tot }}=9-15 \%$ with $\mathrm{d}=1 \mu \mathrm{m}$ and $<<1 \%$ with $\mathrm{d}=10 \mu \mathrm{m}$.

- $\quad \mathrm{PRR}=80 \mathrm{MHz}, \mathrm{T}<3 \mathrm{ps}, \mathrm{W}>0.7 \mathrm{~W}, \mathrm{E}>8.5 \mathrm{~nJ}-\eta_{\text {tot }}=\approx 3 \%$ even with $\mathrm{d}=10 \mu \mathrm{m}$.

A different approach reported in [19] involved a more expensive laser characterized by 4 ps pulse duration (sufficient for a Raman bandwidth of $2-3 \mathrm{~cm}^{-1} @ 532 \mathrm{~nm}$ ), average power $0.5 \mathrm{~mW}$ and PRR $2 \mathrm{MHz}$; it follows that $E=250 \mathrm{~nJ}$. The reported total efficiency for this system is $10 \%$, which could be roughly obtained with $d \approx 35 \mu \mathrm{m}$.

In the literature, a further solution was proposed by exploiting solid state lasers (e.g., Ti:Sa) at $\lambda \approx 800 \mathrm{~nm}$ with $\mathrm{CS}_{2}\left(n_{2}=3.1 \times 10^{-18} \mathrm{~m}^{2} / \mathrm{W}\right)$ as active medium and $T<<10$ ps [18]. Such a device, however, is at the moment much more expensive than the others already described. 
We should also mention systems based on commercial fs lasers [22,33-35] allowing one to achieve time resolution of $\approx 100 \mathrm{fs}$, but with limitations due to the spectral broadening (i.e., spectral resolution is too low for Raman detection) and experimental setups designed for ultrafast, or more generally, pump-probe, spectroscopy [36,37]. The latter represent valuable possibilities to be considered, but they involve high costs and complex optical setups. It is worth noting that this work is focused on a particular application of the Kerr shutters, that is, the TRRS. Hence, issues related to the response time of the involved medium will be not considered because the pulse duration cannot be lowered over the picosecond limit, as already discussed.

Another limit in designing efficient optical Kerr gating systems is represented by the availability of lasers and optical elements. The laser, in fact, should satisfy not trivial technical requirements in terms of PRR and average power, or single pulse energy. Deviations from ideal behavior could be related, for instance, to spectral broadening; inconsistent pulse duration; or PRR, anisotropy and size imperfections of the crystal-imperfect pulse shape.

It is now possible to point out several remarks, assessments and useful considerations for a high-performance setup:

- The efficiency of the setups reported in literature is roughly $10-15 \%$ [17-19,22].

- A setup devoted to TRRS should be based on a gated pulse of short wavelength in order to increase the cross-section.

- $\quad$ A low PRR should be preferred in order to obtain high pulse energy.

- Increasing the length of the active medium $L$ does not lead to exceptional efficiency improvements: In fact, increasing the size could ease the presence of local inhomogeneities, higher absorbance, lower signal strength and problems related to the interfaces between the different slices. The latter happens when several layers of active medium are placed in series.

- $\quad$ Should the laser power exceed $300 \mathrm{~mW}$, many setups are based on gating pulses generated with a laser at 1064 or $808 \mathrm{~nm}$ so that it is easier to find systems with the necessary technical features.

- Working with a large section beam, i.e., $d \approx \mathrm{mm}$, short pulses are needed: $T<<100$ ps. In this case the beam homogeneity represents a relevant issue. A solution could be represented by commercial beam shapers, able to make the beam energy density as constant as possible, e.g., "top hat beam shaping lenses" in order to reach values close to the diffraction limit [38].

\subsection{Uncertainties and Their Consequences in Real Systems}

Let us now describe how the deviations from the ideal behavior of the optical elements and tools can affect the total efficiency. Indeed, the phase factor cannot be always given predicted with absolute confidence, because of random fluctuations of the pulse energy and imperfections of the active medium. By deriving Equation (3), it is possible to obtain the expression of the fluctuations on $\phi$ in order to assess the more relevant term.

$$
\Delta(\phi)=\frac{2 \pi L I}{\lambda} \Delta n_{2}+\frac{2 \pi n_{2} I}{\lambda} \Delta L+\frac{2 \pi n_{2} L}{\lambda} \Delta I+\frac{2 \pi n_{2} L I}{\lambda^{2}} \Delta \lambda
$$

In order to simulate a real system, realistic values were introduced into Equation (8) with their associated errors, and they are listed in Table 3 (a conservative approach was adopted).

A major contribution comes from the term $\frac{2 \pi n_{2} L}{\lambda} \Delta I$, which depends on the beam variability, i.e., spatial and pulse-to-pulse variability. It is worth noting that in Equation (8) only $I$ is considered, since $\phi=\frac{2 \pi n_{2}}{\lambda} L I=\frac{8 n_{2} L W}{\lambda d^{2} T P R R}$ and all the possible sources of error should be considered by introducing $\mathrm{W}, \mathrm{T}$ and PRR. 
Table 3. Values considered for the parameters and their uncertainties.

\begin{tabular}{|c|c|c|c|c|}
\hline Parameter & Symbol & Value & Uncertainty & Notes \\
\hline refractive index & $\mathrm{n}$ & 2.6 & & \\
\hline non-linear refractive index & $n_{2}$ & $8.86 \times 10^{-19} \mathrm{~m}^{2} / \mathrm{W}$ & $10 \%$ & underestimation \\
\hline non-linear medium thickness & $L$ & $10^{-3} \mathrm{~m}$ & $10^{-4} \mathrm{~m}$ & underestimation \\
\hline wavelength & $\lambda$ & $5.32 \times 10^{-7} \mathrm{~m}$ & $6 \times 10^{-10} \mathrm{~m}$ & laser line broadening \\
\hline beam diameter in non-linear medium & $d$ & $1.3 \times 10^{-6} \mathrm{~m}$ & $\mathrm{NO}$ & from beam shaper datasheet \\
\hline pulse duration & $T$ & $88 \times 10^{-12} \mathrm{~s}$ & $\mathrm{NO}$ & - \\
\hline average power & $W$ & $0.34 \mathrm{~W}$ & $\mathrm{NO}$ & - \\
\hline pulse repetition rate & PRR & $8 \times 10^{7} \mathrm{~Hz}$ & NO & $80 \mathrm{MHz}$ (from laser datasheet) \\
\hline average power density & $I$ & estimated from $\mathrm{W}, \mathrm{PRR}, \mathrm{T}, \mathrm{d}$ & $10-50 \%$ & - \\
\hline
\end{tabular}

A further analysis can be performed by simulating the effects of independent fluctuations of the parameters just discussed by exploiting a Monte Carlo model. Random variations were introduced for each parameter and the total efficiency was estimated. The probability distribution around the mean value were considered to be Gaussian with standard deviations equal to those reported in Table 3. The $i$-th term is given by: $X_{i}=X_{i}+$ randn $\cdot \Delta X_{i}$ at each iteration (where randn is a random number with normal distribution with expected value $=0$ and variance $=1$ ). The values reported in Table 3 were used for each $X_{i}$ and $\Delta X_{i}$ with the only exception of $\lambda$; in this case a broader fluctuation was considered, i.e., $6 \times 10^{-8}$, in order verify the behavior of the system over a wider range. For the rotation angle $\theta=45^{\circ}$, the maximum variation $\Delta \theta=0.5^{\circ}$ was considered.

The results, reported in Figure 9, demonstrate that the fluctuations are relevant and mainly due to the term $\Delta I$; indeed, by considering a lower value (e.g., $\Delta I=10 \%$ ) the standard deviation lowers from $90 \%$ to $43 \%$.
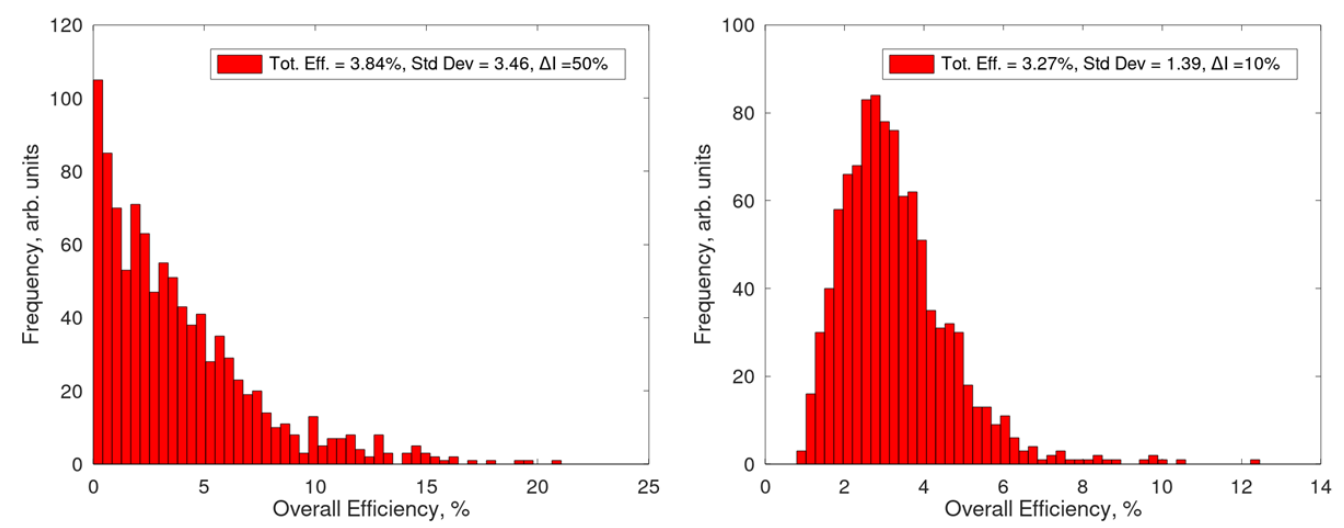

Figure 9. Total efficiency distribution obtained by the Monte Carlo simulation. Maximum fluctuation for $\Delta I: 50 \%$ (left); $10 \%$ (right).

In Figure 10 a transfer function is shown in order to demonstrate the effects of random independent fluctuations (i.e., a Monte Carlo simulation with 100 iterations for each wavelength) of each parameter on the Kerr Gate. This was done by exploiting the values reported in Table 3. 


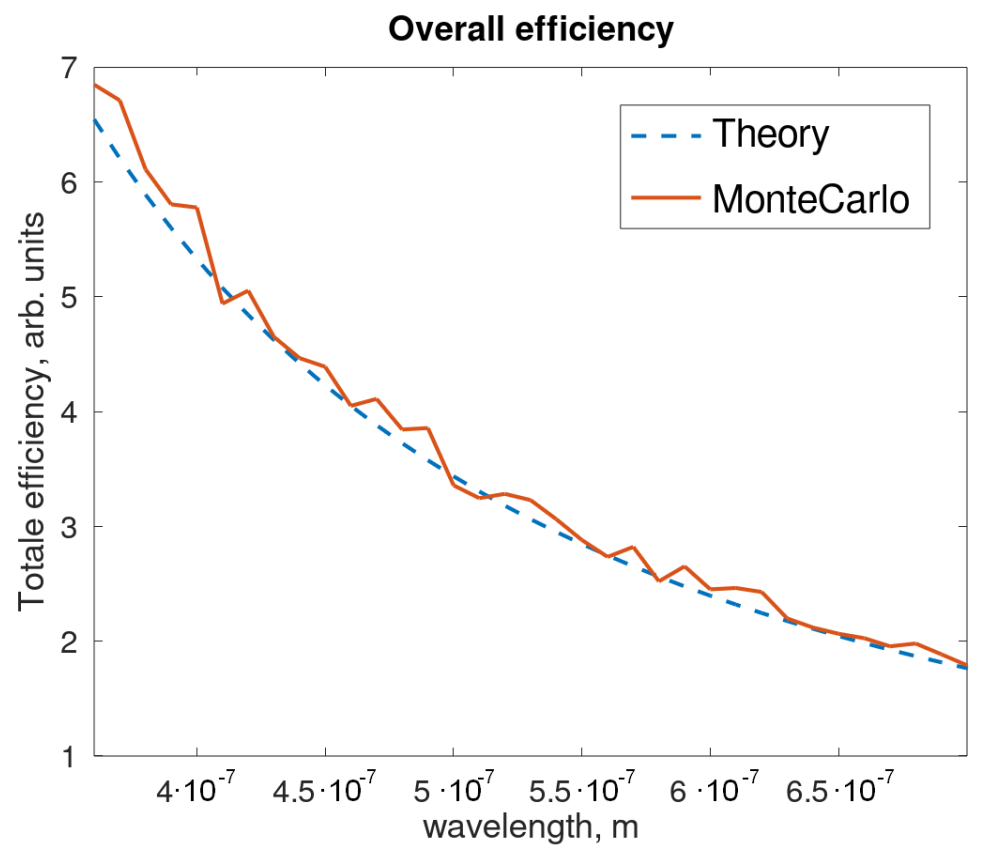

Figure 10. Simulation of total efficiency which could be obtained with a Kerr shutter characterized by the parameters reported in Table 3.

\subsection{Ideal and Real Gate}

To perform Raman spectroscopy, however, a range of different wavelengths should be able to pass through the Kerr cell, and we have seen so far that each choice of the parameters can only allow a perfect gate for a precise wavelength. In order to estimate the performances of a cell over a broad range of wavelengths, a transfer function can be defined from the phase shift at each wavelength. As is intuitive, fluctuations of the parameters will alter this curve from the theoretical expectations.

The transfer function of an ideal setup represents an interesting issue to be explored and characterized for a wide range of wavelengths of the gated pulse. Precisely, a Kerr gate can be considered as ideal when it can induce a phase $\phi=\pi$ for a defined $\lambda$, i.e., $532 \mathrm{~nm}$, so the total efficiency is 1 ; hence this configuration requires a perfect PBS, zero absorbance by the active medium and a perfect angle $\theta$. The other wavelengths will be affected by a different phase factor and the efficiency of the gate will suffer of an evident decay, as reported in Figure 10. In this sense the blue dashed curve can be thought as sort of transfer function that spectrally modulates a characteristic width for the signal passing through the gate.

By introducing random fluctuations of the parameters involved (e.g., by a Monte Carlo model), in an ideal cell, the red curve in Figure 11 (left panel) is obtained. While the theoretical curve oscillates from $0 \%$ to $100 \%$ according to the phase variation of different wavelengths in the non-linear medium, the real total efficiency is restrained to the range $20-90 \%$. In other words, the parameter fluctuations act as a smoothing filter on the transfer function in the wavelength domain.

The fluctuations will introduce a spread in the efficiency for ideal cells too: the distribution that can be obtained in this case is reported in Figure 11 (right panel). When fluctuations are uncorrelated, the optimal efficiency is often reached because of reciprocal compensation; the resulting broadening of the efficiency distribution is not dramatic. 

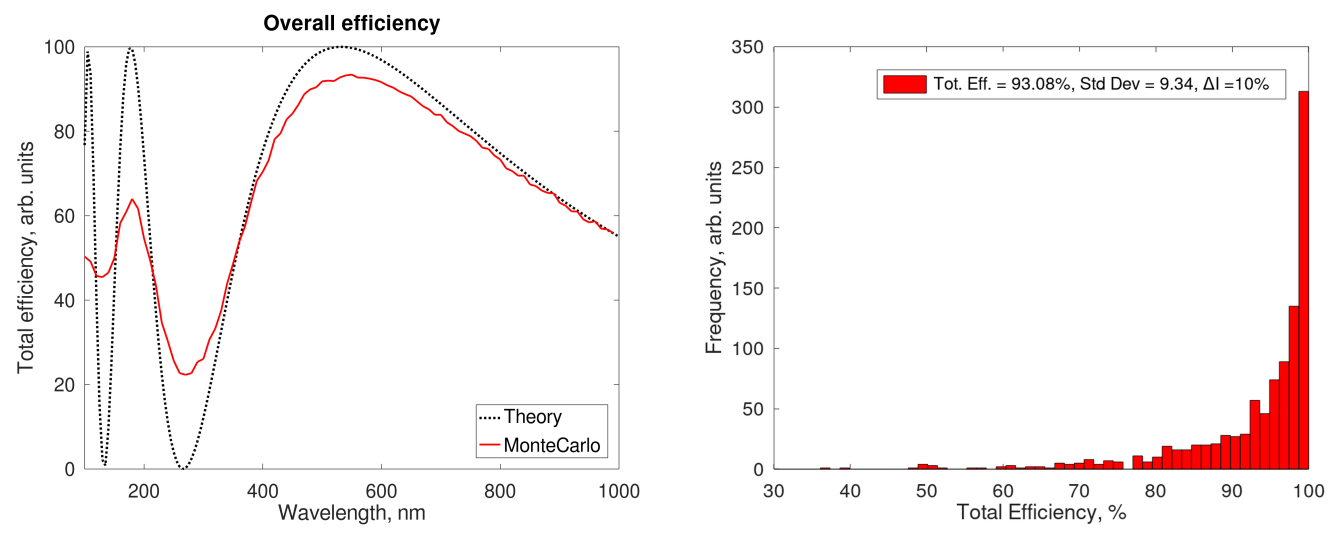

Figure 11. (Left panel) Transfer function of an ideal Kerr gate without (black dashed) and with (red solid) random fluctuations. We considered $1 \mathrm{~mm}$ of $\mathrm{TiO}_{2}$ as active medium, $d=1 \mu \mathrm{m}, T=100 \mathrm{ps}$ ( $\mathrm{E}=23.58 \mathrm{~nJ}$; see Table 2) and the maximum fluctuations reported in Table 3, column "Errors"; (Right panel) total efficiency distribution obtained by a Monte Carlo simulation.

\subsection{Kerr-Gating: Conclusions}

Picosecond time-domain Raman spectroscopy can be experimentally achieved with a Kerr gate, and the fluorescence rejection in measured Raman spectra could be obtained. For instance, a Kerr gate can reach response times as short as $\approx 3$ ps if coupled with $10 \mathrm{~mJ}$, 1 ps excitation pulses at $800 \mathrm{~nm}$ and a repetition frequency of $650 \mathrm{~Hz}$ [39]. However, optical transmittance of the Kerr gate in the open and closed states reaches low efficiencies because of to the incomplete polarization rotation in the Kerr medium and the losses in optical elements. In [17], the realization of an improved Kerr gate with optimized polarizers was reported. Operated with $1 \mathrm{ps}$ pulse laser with at $1 \mathrm{kHz}$ repetition rate, the device reached 4 ps of gating time, and the efficiency of the "open" state was improved to up to $\approx 40 \%$, with an extinction ratio between the open and closed state of $10^{5}$. As a result of that efficient gating, the overall transmission and the collection efficiency of Raman light reached up to 1.6 times and 1.7 times, respectively, relative to their earlier results [39]. However, due to the limitations described so far, the Kerr gate method has not been widely used. The necessity of high-energy pulsed light, in fact, easily makes this method non-compliant with safety regulations, since photo-induced damage risk is increased [11].

\section{Time-Gated Single Photon Avalanche Photodiode (TG-SPAD) \\ 4.1. Introduction and Theory}

SPADs have been extensively explored for the detection of low intensity light. The implementation of SPADs in complementary metal-oxide-semiconductor (CMOS) technologies has boosted the application of the CMOS SPADs, owing to their capability of being integrated with CMOS control electronics [40-42]. With the abilities of single photon detection and a sub-nanosecond temporal response, the CMOS SPAD has been used in various applications involving the time-correlated single photon counting (TCSPC) and time-gated detection applications, including fluorescence lifetime imaging (FLIM) [43,44], superresolution FLIM [45], fluorescence fluctuation spectroscopy (FFS) [46], near infrared spectroscopy (NIRS) [47,48], light detection and ranging (LIDAR) and 3D time-of-flight [49-51], quantum mechanics [52], quantum communication [53] and quantum imaging [54,55]. A SPAD can be operated in either free running or time-gated (TG) modes. The fast gating capability makes the CMOS SPAD a low-cost alternative to ICCDs and a solution to be compared with an optical Kerr gate. Recently, CMOS TG-SPADs have been proposed for fluorescence rejection in Raman spectroscopy [56-59], where external pulse generators allowed one to control the detection system and sub-nanosecond windows were achieved.

A SPAD is essentially a p-n junction (see Figure 12) biased above the avalanche breakdown voltage (VBR), the so-called Geiger mode. Controlled by an external quenching circuit, the single photon detection process of the SPAD can be divided into four phases. 
These are carrier generation by a photon, internal carrier multiplication, quenching of the voltage across the SPAD and recharge. Figure 13 shows the four phases involved in a photon detection process on the I vs. V characteristic curve of the SPAD.

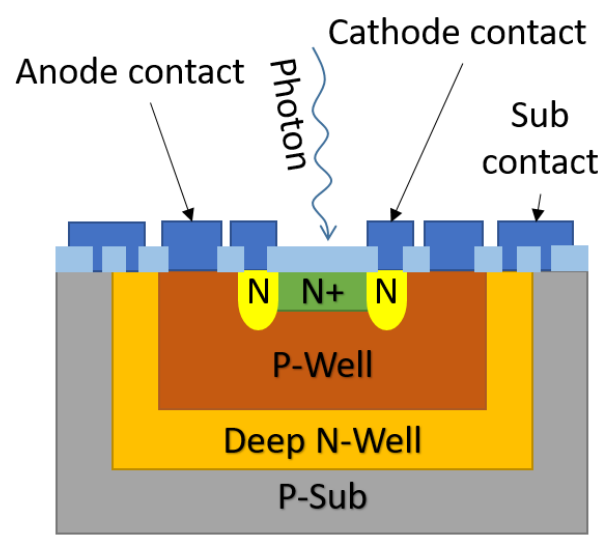

Figure 12. The junction scheme of a single photon avalanche photodiode (SPAD) device.

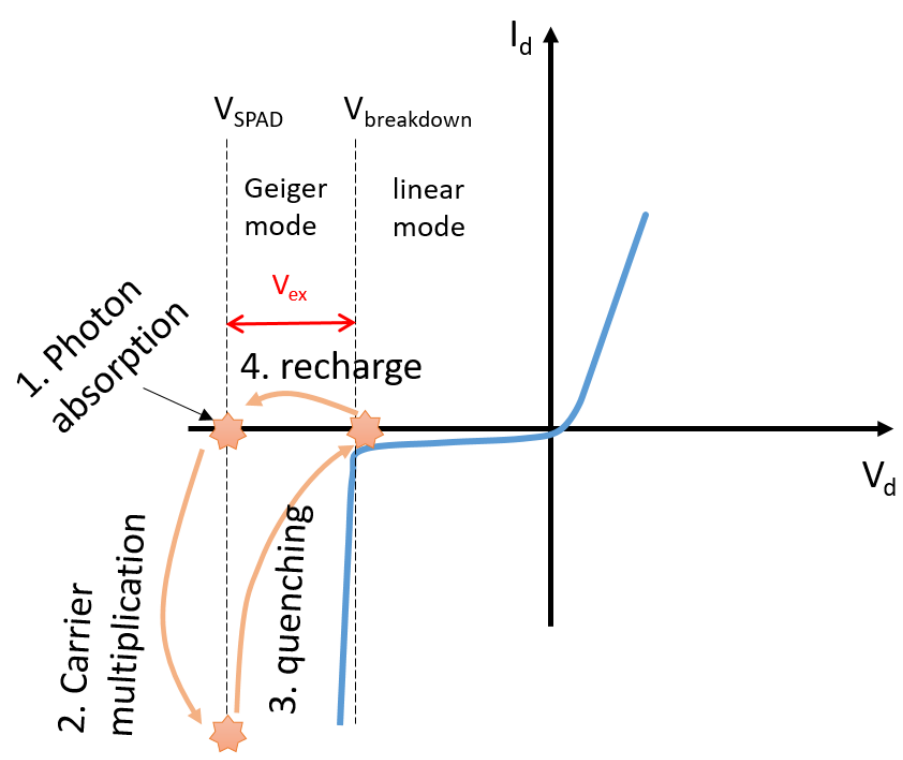

Figure 13. Current-voltage (IV) characteristic curve of an avalanche photodiode operated in Geiger mode.

Before photon detection, the SPAD is biased above the VBR, and this overdrive voltage is named the excess bias $\left(V_{e x}\right)$. The detection cycle of the SPAD begins when an absorbed photon results in the generation of an electron-hole pair of free carriers. In the second phase, the photon-generated carriers are accelerated by the high electric field and multiplied in the depletion region. As discussed in [60], basically, two processes are involved in this phase. The first process refers to impact ionization induced by the high electric field, which triggers the carrier multiplication process that creates an avalanche of secondary carriers. The ionization probability strongly depends on the electric field, which can be adjusted by applying different $V_{e x}$. The second process is internal quenching. The ionization process increases the local current density, which causes a larger voltage drop across the space charge resistor, and in turn weakens the ionization process. When the two processes are balanced, a current pulse in the $\mathrm{mA}$ range is then delivered to the external control circuit, which senses and quenches the avalanche.

The fast quenching of the avalanche process is important for the operation of the SPAD, because it protects the diode from overheating and related damages. After being fully quenched, the SPAD is recharged back to the Geiger mode. The entire detection cycle 
is shown in Figure 13. The external control circuit plays a dominant role in the detection cycle. The SPAD control circuit is designed to match a specific application. For instance, the SPAD can be designed in a pixel array or as a discrete component, operated in free running or time-gated mode. Each SPAD application has different requirements, and the quenching and recharge circuits of the SPAD must be designed accordingly.

The parameters commonly used to characterize the performance of a SPAD are described next.

1. Dead time: As depicted in Figure 13, during the time when a SPAD undergoes photon absorption, carrier multiplication, quenching and recharge, the SPAD is not able to detect a subsequent photon. Dead time is defined as the time it takes to complete a detection cycle, which determines the maximum counting rate of a SPAD. Therefore, fast quenching and recharge circuits are required for high-speed applications.

2. Dark count rate (DCR): DCR is defined as the number of counts per second when the SPAD is in the dark. A major source of DCR in a CMOS SPAD at room temperature is the thermal generation of free carriers, and the generation rate is also related to the ionization probability. Therefore, DCR depends on temperature, excess bias and the size of the active area of the SPAD. DCR determines the minimum incident photon rate that can be detected.

3. Photon detection efficiency (PDE): When a light source with certain intensity is incident on the detector, only a portion of the incident photons can be detected due to several reasons, including surface reflection, photon absorption before reaching the depletion region, absorption coefficient of the material and the probability of triggering an avalanche. PDE is defined as the ratio of the number of voltage pulses detected to the number of total incident photons. PDE is the most important parameter to evaluate the detection efficiency of a SPAD, and it is a function of the incident wavelength and excess bias. The PDE of a SPAD corresponds to the external quantum efficiency of other types of photodetectors.

4. Afterpulsing probability (AP): During the quenching phase, a large number of carriers flow through the depletion region. Some carriers are trapped in deep energy levels within the depletion region and released later to trigger a second detection cycle that is not initiated by photon absorption. The pulses generated by the released carriers from traps are named afterpulsing in SPAD. AP is correlated to the avalanche current and its duration, and thus, to the amount of charge of the avalanche pulse. Therefore, AP is proportional to the parasitic capacitance of the photodiode and the quenching time. The latter can be optimized by specially designed external control circuits.

5. Fill factor (FF): To achieve fast quenching and resetting, complex control circuits are designed for SPAD pixels. In active SPAD pixels, the area of the control circuits is often comparable to or larger than the optically active area of the SPAD. Fill factor is defined as the ratio of the SPAD active area to the total pixel area. For better usage of the chip area, a high FF is preferred.

6. Timing skew (TS): The shot noise of the events just described (i.e., parameters 1-5) contributes to distorting the spectra measured with time-resolved Raman spectrometers employing CMOS SPAD line sensors, and the so-called timing skew of the sensors [61-63]. Due to their spatial extension, a timing mismatch happens among the pixels of the sensor, i.e., the points of the spectrum. This leads in turn to shifted time gates for each pixel, so that photon counts at different spectral points may vary if the signal varies in time. This kind of sampling error can further distort the spectra.

DCR and PDE are strongly determined by the fabrication process, although some freedom is available for the designs of the control circuits in SPAD pixels. Optimized designs of quenching and recharge circuits mostly target reduction of the dead time and $\mathrm{AP}$, and increase in FF. Two types of SPADs control circuits will be discussed: free running and time-gated. 


\subsection{Free Running Operation}

"Passive quenching and recharge" should be the simplest approach for the design of a SPAD in free running mode. An example of a possible circuit to be used in this case was discussed in [64]. It consists of a quench resistor $\left(R_{Q}\right)$ connected in series with the photodiode which is biased at $\left(V_{B R}+V_{e x}\right)$. To efficiently quench the avalanche current in a very short time, $R_{Q}$ is very large, usually in the range of $\mathrm{k} \Omega$ to $\mathrm{M} \Omega$. Consequently, even a low avalanche current can result in a high enough voltage drop across $R_{Q}$. This voltage drop forces the bias of the SPAD to reduce below the breakdown voltage $V_{B R}$ and then quench the avalanche process. The time it takes to fully quench the avalanche current is determined by $R_{Q}$, and the dynamic resistance $\left(R_{D}\right)$ and capacitance $\left(C_{D}\right)$ of the diode.

Figure 14a shows a simple SPAD circuit with passive quenching and recharge [64]. The equivalent quenching and recharge circuits are given in Figure $14 \mathrm{~b}, \mathrm{c}$ respectively. The external biases are applied to the anode $\left(V_{A}\right)$ and the quench resistor $\left(V_{C}\right)$, and photon detection is sensed from the variations of the cathode potential $\left[V_{O}(t)\right]$. With this bias condition, the excess bias equals $V_{e x}=V_{C}-V_{A}-V_{B R}$.

By applying the Kirchhoff's law to the passive quenching and recharge phases (Figure 14b,c), it is possible to estimate the time constants characterizing the processes:

$$
\begin{gathered}
\tau_{\text {quench }}=\frac{C_{D} R_{D} R_{Q}}{R_{D}+R_{Q}}, \frac{C_{D} R_{D} R_{Q}}{R_{D}+R_{Q}}=R_{D} \| R_{Q} \\
\tau_{\text {recharge }}=C_{D} R_{Q}
\end{gathered}
$$

The quench resistor $\left(R_{Q}\right)$ dominates the recharge time, and the quenching time is correlated with the parallel combination of $R_{Q}$ and $R_{D}$. Since $R_{D}$ is much lower than $R_{Q}$, the quenching time is shorter than the recharge time: $10^{-1} \mathrm{~ns}$ vs. tens of nanoseconds.
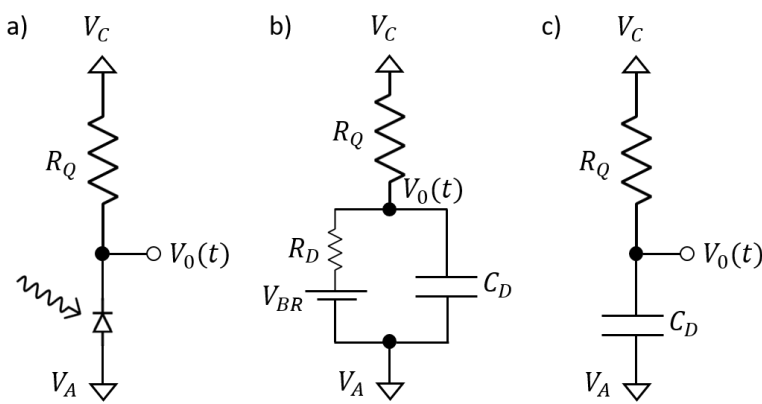

Figure 14. (a) A SPAD with passive quenching and recharge circuits; (b) equivalent circuit of passive quenching; (c) equivalent circuit of passive recharge.

In a passive quenching circuit, the quench resistor usually occupies an area smaller than the area of the photodiode, so a high FF can be achieved. Passive quenching is also suitable for the design of large pixel arrays. A drawback of the passive quenching circuit is the unstable dead time. If a photon arrives during the slow recharge phase, since the SPAD is biased at the Geiger mode, this photon could trigger a second detection and stop the recharge process. As a result, the dead time is expanded becoming longer than the designed one.

Another limitation of the passive quenching circuit is the AP. As mentioned above, AP is related to the quenching time and the parasitic capacitance. Since the circuit only contains a resistor and a photodiode, the circuit's capacitance mainly comes from the capacitance of the photodiode. The capacitance of the photodiode is determined by the fabrication process, the SPAD area and reverse bias voltage. For SPADs implemented in deep sub-micron CMOS technologies, the capacitance is small and in the range of $\mathrm{fF}$. This is an advantage of the passive quenching circuit, but the quenching time is long enough for carriers to be trapped. If the SPAD is in the Geiger mode, then trapped carriers can be released later, leading to afterpulsing. In addition, the captured carriers released during 
the long recharge phase can cause the dead time to increase. To solve the dead time and afterpulsing problems, high-speed control circuits are designed to reduce the quenching and reset times of the SPAD.

A possible improvement is represented by a mixed passive-active quenching circuit [65]. In this case, the dead time of the SPAD is effectively shortened.

Therefore, high counting rates can be achieved for SPADs with active quenching and recharge. As given in [66], a maximum counting rate of $185 \mathrm{MHz}$ was achieved with a mixed passive-active quenching circuit, corresponding to a dead time of 540 ps. Regarding the AP, the parasitic capacitance at the node of the SPAD cathode was increased because of the complex connection, but the quenching time was also reduced. Further solutions were implemented in order to reduce the AP (e.g., to add a hold-off time). However, the FF of the mixed quenching circuit is low, since complicated control circuits are used. Hence, SPAD pixels with passive quenching and reset are still the preferred choice for design of large arrays.

The free running SPADs have been widely used in the time-resolved applications with the TCSPC technique, such as in FLIM and NIRS. Regarding Raman spectroscopy applications, the time-gated SPADs are more suitable due to its fluorescence suppression capability.

\subsection{Time-Gated Operation}

The quenching and recharge circuits reviewed in the previous sub-section were mainly developed for free running SPADs $[65,67]$. However, there are applications in which the photon detection is required only for a short time window after a pulse excitation, and the time window has to be precisely synchronized with the excitation, as shown in Figure 15. This windowed mode of photodetection is known as the time-gated operation of SPAD. Typical examples requiring time-gated photodetection include Raman spectroscopy, to remove the fluorescence, and NIRS. To operate a SPAD in time-gated mode, the control circuit is crucial, because it quickly gates the SPAD above and below the avalanche breakdown voltage and synchronizes the detection with the pulse excitation.

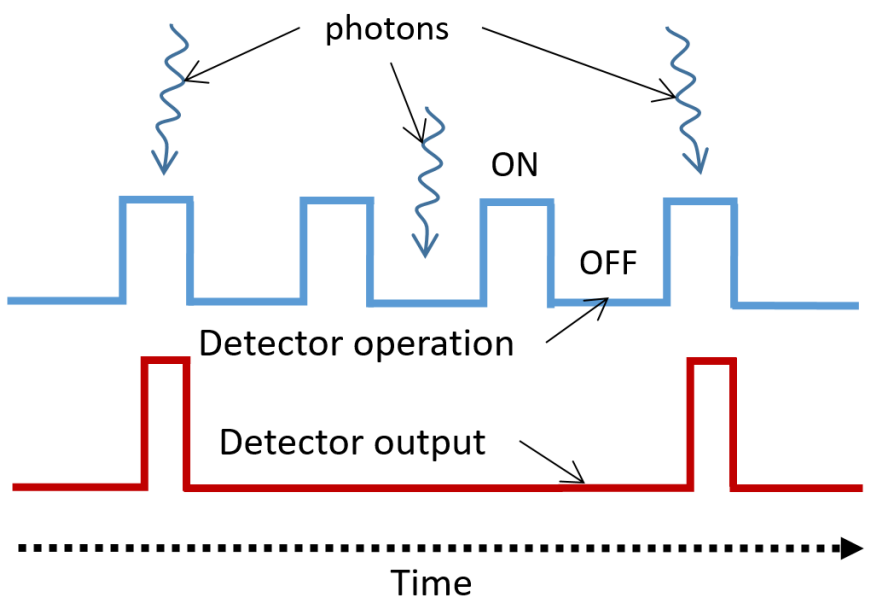

Figure 15. Time-gated operation.

In [64], a common way to apply the gating signal is described; see Figure 16a. A DC pre-bias is provided by a voltage source $V_{C}$ and the gating signal is coupled through the capacitor $C_{1}$. To achieve fast gating, $C_{1}$ should be small. However, to maintain long enough pulse duration, $C_{1}$ cannot be too small. Thus, the design of the input $A C$ coupling network is important for appropriate gating and sensing of the SPAD.

To sense an avalanche occurring during the narrow gate, usually an AC pick-up circuit is applied and its output is fed to a high speed comparator. 
Upon detection, the avalanche current flows through the resistors $R_{3}\left(R_{2}>>R_{3}\right)$, which quickly quenches the avalanche. Then, the SPAD is brought back to the Geiger mode by $R_{2}$. The function of the AC pick-up network is to sense the transient voltage increase of the SPAD's anode that is induced by the avalanche current. However, because of the large $R_{2}$, a wide gate window is required, and this limits the SPAD's maximum counting rate.

On the other hand, there are voltage spikes occurring during voltage transitions. As shown in Figure 16b, the SPAD is biased above and below the breakdown voltage. The fast voltage transition introduces a sudden variation to the anode voltage, and spikes (rising and falling) appear at the output of the pick-up network, as shown in Figure 16c. To reduce the effect of the spikes, the threshold $\left(V_{T H}\right)$ of the comparator should be higher than their amplitude. Since the amplitude of the voltage spike is related to the transition speed and amplitude of the gating signal, the spike's amplitude can be higher than the avalanche pulse. In these situations, a high value for $V_{T H}$ is not an efficient choice.
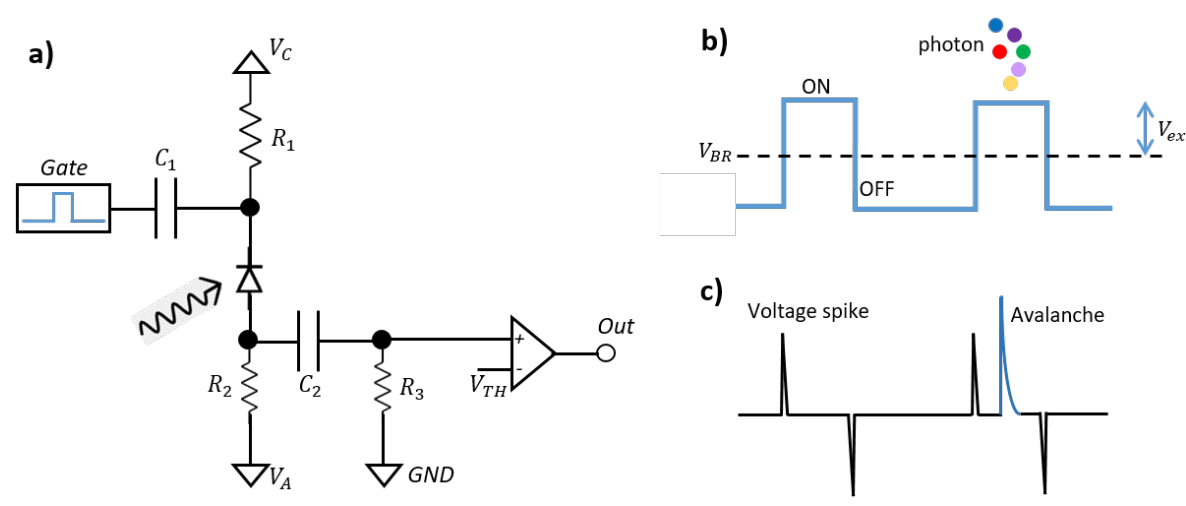

Figure 16. Time-gated operation scheme. (a) A basic diagram of a SPAD with a time gated control circuit. (b) A timing diagram of the gating signal; (c) a timing diagram of the output of the AC pick-up circuit.

\subsection{Raman}

In this sub-section, several experimental approaches will be discussed in order to review the different experimental setups based on the SPADs that have been proposed in the literature and could be implemented for time-gated Raman spectroscopy.

Most of the papers proposed several versions of the same idea, which is to introduce arrays of SPADs characterized by an increasing size. This paper is not aimed at a detailed review of these systems, which has been recently published [68] (see Table 4 for an updated summary); hence we will focus only on the descriptions of some relevant examples. This information will be used to compare this approach with the others here presented.

Sensors based on a SPAD array were employed for imaging in several papers, and a relevant step towards in this field is represented by the experiment described in [69]; further examples are reported in [41,43,70]. A time-gated $128 \times 128$ CMOS SPAD array was triggered by a small fraction of the excitation laser; this optical trigger was detected by a single SPAD to convert the optical into an electrical signal. An external delay line was employed to synchronize the SPAD activation in order to optimize the measurement of the Raman spectrum on the sensor plane. This system was used to perform time gated FLIM and TRRS. 
Table 4. SPAD: state of the art, according to [56,59,68,71]. The column Technology reports a number in (nm), and acronyms refer to specific semiconductor manufacturing processes and design rules employed to fabricate each SPAD. HV: highvoltage process; CIS: CMOS imaging sensor process; 3D: 3D integration technology (usually backside illuminated—BSI).

\begin{tabular}{|c|c|c|c|c|c|c|c|}
\hline Sensor & Year & SPAD Array & Technology [nm] & Pixel Pitch $[\mu \mathrm{m}]$ & Fill Factor [\%] & PDE [\%] & DCR $\left[\mathrm{cps} / \mu \mathrm{m}^{2}\right]$ \\
\hline [72] & 2003 & $8 \times 4$ & 800 & - & $<1$ & 0.2 & 1.6 \\
\hline [73-79] & 2007 & $8 \times 1$ & - & 198 & 5 & 2.5 & 1.0 \\
\hline$[80,81]$ & 2007 & $64 \times 64$ & $350 / \mathrm{HV}$ & 40 & $<1$ & 0.1 & 71.0 \\
\hline [82-84] & 2008 & $128 \times 128$ & $350 / \mathrm{HV}$ & 25 & - & - & 17.0 \\
\hline$[85,86]$ & 2009 & $4 \times 4$ & $350 / \mathrm{HV}$ & 36 & $<1$ & - & 1.0 \\
\hline [87-89] & 2009 & $60 \times 48$ & $350 / \mathrm{HV}$ & 85 & $<1$ & 0.1 & 7.0 \\
\hline [90-93] & 2009 & $32 \times 32$ & $350 / \mathrm{HV}$ & 100 & 3.1 & 1.3 & 12.7 \\
\hline$[43,94-104]$ & 2009 & $32 \times 32$ & $130 / \mathrm{CIS}$ & 50 & 1 & 0.4 & 4.0 \\
\hline [44] & 2009 & $64 \times 4$ & $350 / \mathrm{HV}$ & 26 & 34 & 10.9 & 4.6 \\
\hline [105-108] & 2009 & $32 \times 32$ & $350 / \mathrm{HV}$ & 30 & 3.1 & 1.1 & 5.0 \\
\hline [109] & 2009 & $7 \times 2$ & $350 / \mathrm{HV}$ & - & - & - & 13.0 \\
\hline$[110,111]$ & 2011 & $128 \times 128$ & $350 / \mathrm{HV}$ & 25 & - & - & 6.6 \\
\hline$[112,113]$ & 2011 & $160 \times 128$ & $130 / \mathrm{CIS}$ & 50 & 1 & 0.3 & 2.0 \\
\hline [114-116] & 2011 & $32 \times 32$ & $350 / \mathrm{HV}$ & 25 & 20.3 & - & 5.4 \\
\hline$[117,118]$ & 2012 & $32 \times 32$ & $350 / 3 \mathrm{D}$ & 50 & 75.4 & - & 39.7 \\
\hline [119-121] & 2012 & $32 \times 32$ & $130 / \mathrm{CIS}$ & 22 & 10 & - & 13.7 \\
\hline$[122,123]$ & 2013 & $64 \times 64$ & 130 & 48 & $<1$ & 0.3 & 28.0 \\
\hline [124] & 2013 & $416 \times 4 \times 4$ & $350 / \mathrm{HV}$ & $30 \times 50$ & 55.6 & 17.0 & 39.0 \\
\hline$[56,125]$ & 2013 & $1024 \times 8$ & $350 / \mathrm{HV}$ & 24 & 44.3 & 9.6 & 29.0 \\
\hline$[57,58,126-128]$ & 2013 & $128 \times 8$ & $350 / \mathrm{HV}$ & 33 & 23 & 5.8 & 71.0 \\
\hline [129-132] & 2013 & $720 \times 16 \times 8$ & $130 / \mathrm{CIS}$ & 19 & 42.9 & 12.0 & 6.2 \\
\hline [70] & 2013 & $10 \times 43$ & $350 / \mathrm{HV}$ & $20 \times 100$ & 67 & 4 & - \\
\hline [133-138] & 2014 & $512 \times 128$ & $350 / \mathrm{HV}$ & 24 & - & - & 12.0 \\
\hline$[59,64]$ & 2014 & 1 & 130 & 10 & 9.8 & 3 & - \\
\hline$[51]$ & 2014 & $32 \times 32$ & 350 & 150 & 3.14 & $20-55 \%$ & $120 \mathrm{cps}$ \\
\hline [139] & 2015 & $416 \times 18 \times 9$ & $350 / \mathrm{HV}$ & $30 \times 50$ & 57 & 18.6 & 43.0 \\
\hline$[140,141]$ & 2015 & $256 \times 2$ & $130 / \mathrm{CIS}$ & 24 & 43.7 & - & 5.4 \\
\hline [142] & 2015 & $256 \times 256$ & $130 / \mathrm{CIS}$ & 8 & 19.6 & - & 4.0 \\
\hline$[143,144]$ & 2015 & $400 \times 1$ & $130 / 3 \mathrm{D}$ & 11 & 23.3 & 2.8 & 357.0 \\
\hline [145] & 2016 & $128 \times 120$ & $65 / 3 \mathrm{D}$ & 8 & 45 & 12.4 & 36.2 \\
\hline [146] & 2016 & $72 \times 60$ & 180 & 35 & 14.4 & 0.4 & 2.3 \\
\hline$[147,148]$ & 2016 & $256 \times 1$ & $350 / \mathrm{HV}$ & 24 & 40 & 13.6 & 11.0 \\
\hline [149] & 2016 & $160 \times 120$ & $350 / \mathrm{HV}$ & 15 & 21 & - & 12.0 \\
\hline [150-152] & 2016 & $320 \times 240$ & $130 / \mathrm{CIS}$ & $8 / 16$ & - & - & 3.0 \\
\hline [153] & 2017 & $1024 \times 16$ & $130 / \mathrm{CIS}$ & 24 & 49.3 & - & - \\
\hline$[61,62]$ & 2017 & $256 \times 16$ & $350 / \mathrm{HV}$ & 35 & 26 & - & - \\
\hline [154] & 2017 & $256 \times 8$ & $130 / \mathrm{CIS}$ & 24 & 43.7 & - & 5.4 \\
\hline$[50,155-157]$ & 2017 & $32 \times 32$ & 180 & 28 & 28 & 13.4 & 0.6 \\
\hline [158-160] & 2017 & $512 \times 512$ & 180 & 16 & 10.5 & 5.2 & 0.3 \\
\hline [161] & 2018 & $256 \times 256$ & $130 / \mathrm{CIS}$ & 16 & 61 & - & 51.0 \\
\hline [162] & 2019 & $400 \times 400$ & $65 /$ CIS & 6 & 70 & - & $100 \mathrm{cps}$ \\
\hline [163] & 2019 & $256 \times 256$ & $40 / 90 / 3 \mathrm{D}$ & 9.2 & 51.0 & 11.7 & 20 cps \\
\hline$[71,164]$ & 2020 & $1024 \times 1000$ & 180 & 9.4 & $7.0 / 13.4$ & $0.7 / 3.6$ & $0.4 / 2.0 \mathrm{cps}$ \\
\hline
\end{tabular}

In [63] the spectrometer included a time-resolved, $16 \times 256$ line sensor, with an integrated 256-channel 3-bit on-chip time-to-digital converter (TDC). The SPAD array consisted of 16 columns and 256 rows. Each one of the 256 columns contained 16 SPADs and determined the signal in a single point of the Raman spectrum, providing a final spectrum of 256 points covering the wavenumber range of approximately $1600 \mathrm{~cm}^{-1}$. A suitable diffraction grating was employed to separate the different wavelengths with a final resolution of $\approx 6 \mathrm{~cm}^{-1}$. Excitation was provided by a $532 \mathrm{~nm}$ pulsed laser (pulse width: $160 \mathrm{ps}$; bandwidth: $0.11 \mathrm{~nm}$; PRR: $350 \mathrm{kHz}$; maximum pulse energy: $1 \mu \mathrm{J}$ ). The SPAD was biased to the Geiger mode (i.e., single photon detection) by compensating the delay between the optical and electrical signals. This was achieved by sending a logic level trigger signal, generated 
by the optical detector, to the CMOS SPAD after the transition into an off-chip digital delay unit. The Geiger mode was activated only for short intervals; hence, the Raman signal was detected while the fluorescence photons were rejected. The arrival time of the photons was measured by an integrated on-chip 256-channel, 3-bit TDC with a resolution of $\approx 50$ to $\approx 200 \mathrm{ps}$. The final time gate width was chosen in the data post-processing phase by selecting the analyzed time bins. The system was equipped also with a 3D mechanical stage to move the sample and a field-programmable gate array control circuit (FPGA) to drive the sensor circuit.

A fluorescence-suppressed Raman spectrometer can be employed for several applications. In [165] it was used to obtain the chemical imaging of human teeth. The experimental setup was based on a $16 \times 256$ time-resolved CMOS SPAD with an integrated 256-channel, 3-bit on-chip TDC converter [62,63]. A simple unsupervised machine learning algorithm, i.e., k-means clustering, was introduced to obtain high quality images which were compared with those achievable with commercial systems. The time-resolved spectra had a 4.4-8.8 times higher signal to peak-to-peak noise ratio by considering the same radiant exposure $\left(\approx 300 \frac{\mathrm{J}}{\mathrm{mm}^{2}}\right)$ for both the spectrometers. The excitation source was a pulsed laser (wavelength: $532 \mathrm{~nm}$; bandwidth: $0.11 \mathrm{~nm}$; pulse width: $160 \mathrm{ps}$; pulse energy: $0.5 \mu \mathrm{J}$; PRR: $350 \mathrm{kHz}$ ). Additionally, in this case, the SPAD was biased to the Geiger mode as described in the previous paragraph. Some of the laser pulses were sent to an optical detector in order to synchronize the Geiger mode with the excitation source. The detection setup was completed by a diffraction grating (final resolution: $\approx 6 \mathrm{~cm}^{-1}$ ) and an FPGA suitably programmed to drive the electronics involved.

In [140] the integrated TDC converters performed pre-calculations using using centerof-mass method (CMM). The sensor was characterized by a timing of 65,000 photons/pixel at $200 \mathrm{~Hz}$ line rate at $40 \mathrm{ps}$ resolution. The array was composed by $256 \times 2$ SPADs and equipped with a TCSPC for each pixel which could generate histograms with $320 \mathrm{ps}$ bin resolution. The array was characterized by two lines: the first one was optimized for detection of wavelength range $450-550 \mathrm{~nm}$ and named the blue line, and the second one was optimized for 600-900 $\mathrm{nm}$ and named the red line. The latter was designed to obtain higher detection efficiency and lower noise. In this work, two remarkable novelties were introduced: the pixels had individual TCSPC electronics and the spectrometer SPAD array had computational logic for the CMM calculation of photon arrival times on the fly. Parallelized TCSPC can be useful in low light circumstances. It can act as a measurement tool for fluorescence decays and time gating by post-processing the acquired TCSPC histograms. The CMM estimation improves photon detection efficiency, since in this case, it is not necessary to transfer TDC codes from the array to the related electronics.

Commercial TCSPC systems are usually single-channel systems. More complex devices, such as 8 channel [166] or 16 channel [167] TCSPC show several drawbacks since they are not fully independent. Indeed, at high count rates, the high probability of coincidence or cross-talk could have negative consequences on their performances.

In [168], a laser microchip pumped by $808 \mathrm{~nm}$ CW laser was employed (pulse duration: $100 \mathrm{ps}$; pulse energy at $1064 \mathrm{~nm}$ : >20 nJ; PRR: up to $500 \mathrm{kHz}$ - variable according to the pump energy). As in most DPSS lasers, the narrow linewidth $(<0.1 \mathrm{~nm})$ was appropriate for Raman spectroscopy.

The pump was a diode laser, stabilized in wavelength $(1064 \mathrm{~nm})$, single-mode and fiber-coupled. A $40 \mu \mathrm{m}$ FWHM Gaussian spot was focused on the laser microchip, and its output was collimated and sent through a miniaturized optical insulator to cut off back reflections. Light was then focused into a MgO:PPLN crystal, obtaining the harmonic at $532 \mathrm{~nm}$ with $60 \%$ conversion efficiency; collimated again; and the remaining $1064 \mathrm{~nm}$ light was removed using a short-pass filter. Significant drawbacks of this setup were the limited lifetime of the saturable absorber output coupler and the power of the pump laser. In fact, to minimize the risk of laser failure, the laser PRR was set significantly lower than its maximum capability. 
The detector was a $1024 \times 8$ pixel SPAD array, well described in [56]. This SPAD array was designed and fabricated employing standard CMOS processes and features. The signal exiting the spectrometer was aligned so that about $90 \%$ fell in the two center rows of the SPAD, which actually operates as a $1024 \times 2$ array. Furthermore, the readout architecture was modified to operate the SPAD detector up to $1 \mathrm{MHz}$. A fast FPGA was used to accumulate the counts of each pixel over a large number of gates, and subsequently transfer these data at a slower rate to the computer. Excitation was provided by a $532 \mathrm{~nm}$ pulsed laser (pulse width: $400 \mathrm{ps}$; PRR: $1 \mathrm{kHz}$; pulse energy: $20 \mathrm{~J} /$ pulse). Due to the low laser repetition rate, the afterpulsing effect was negligible since the sensor had a dead time of $1 \mathrm{~ms}$ to release previously created trapped carriers. The laser light was used to trigger the detector, and a the optical signal was converted into an electrical one by exploiting a single pixel SPAD.

In [169] an optical spectrometer was built around a SPAD based 512 pixel line sensor. The excitation source uses a $532 \mathrm{~nm}$ pulsed laser diode, with a $4 \mathrm{kHz}$ repetition rate, peak power of $6 \mathrm{~kW}$, a pulse width of $0.6 \mathrm{~ns}$ and a spectral broadening line width narrower than $0.1 \mathrm{~nm}$.

The CMOS sensor was a 16.5 giga-events/s $1024 \times 8$ SPAD line sensor with $23.78 \mu \mathrm{m}$ pixel pitch. Every pixel has a 32 bin histogramming time-to-digital converter (TDC) with zoomable time ranges from 1.6 up to $204.8 \mathrm{~ns}$. The sensor can operate in three main modalities:

1. Single photon counting (SPC) mode (with a throughput of 65 giga-events/s);

2. Time-correlated single photon counting (TCSPC) mode (194 million-events/s);

3. Histogramming mode (HistMode) (16.5 giga-events $/ \mathrm{s})$.

The histogramming mode generates on-chip TCSPC histograms at a per-pixel level to avoid a readout of raw TCSPC events, yielding up to two orders of increase in SPAD photon processing rates, enabling fast scanning or low-I/O-power, time-resolved spectroscopic imaging applications.

A completely different approach was introduced in $[59,64]$, where a portable low-cost Raman spectrometer based on a concave grating and a single time-gated CMOS SPAD was designed. In this case the detector was moved and the measurement was not contextual for all the wavelengths; hence it takes longer than the setup previously described (see Figure 17 for the scheme of the proposed setup).

Unlike [56,58], in this case the SPAD was gated using on-chip pulse generators with a fixed gate window of $3.5 \mathrm{~ns}$, which offered a minimum detection windows of $\approx 200 \mathrm{ps}$. The temporal resolution of the TG-SPAD was measured to be $\approx 60$ ps with a very short laser pulse.

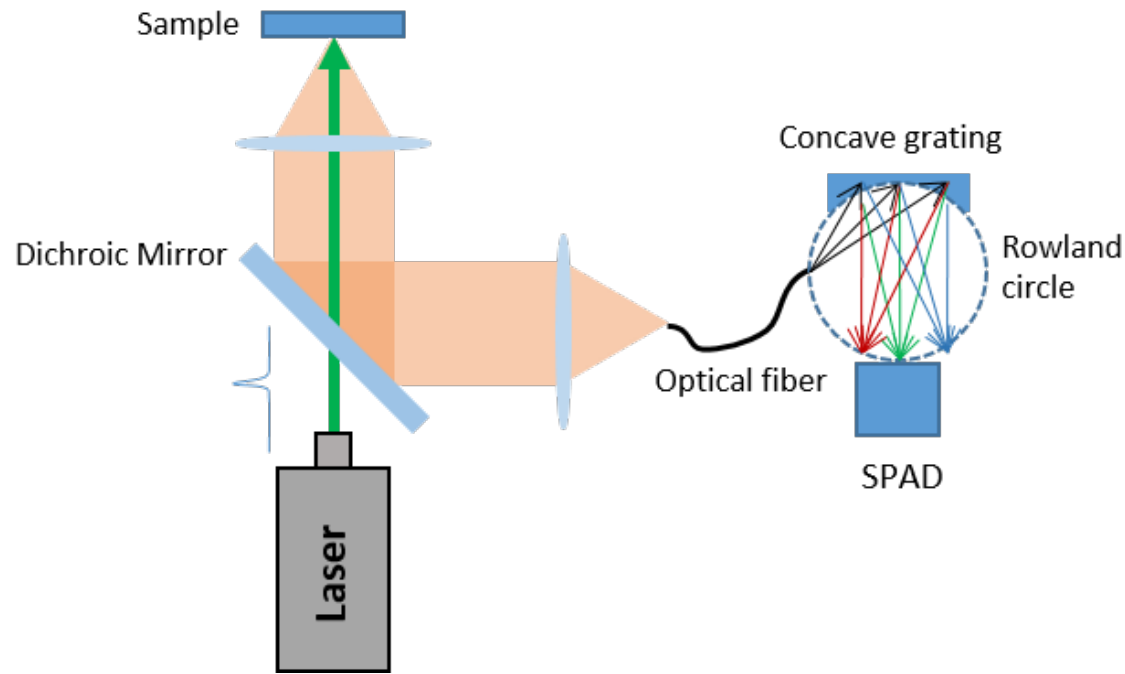

Figure 17. Optical diagram of the proposed TG Raman spectrometer reported in [59]. 
An optical fiber collects the signal towards the Rowland circle of the concave grating, as shown in Figure 17. In this configuration, the Rowland circle is defined as a circle characterized by a diameter equal to the distance between the grating and the SPAD surface which shares part of the circumference with the concave grating. By placing the entrance slit on the Rowland circle, different wavelengths will be focused in different points of the circumference. The whole spectrum can be acquired by moving a detector along the circle, but it is worth noting that focal plane of the Rowland circle is curved, while the active surface of the SPAD is planar. It follows that the whole SPAD area cannot be focused; hence the spectral resolution and the quality of the measurement are limited.

A $532 \mathrm{~nm}$ solid state laser was employed in this setup. The concave grating was optimized for the characterization of biological samples showing Raman shifts in the range of $500 \mathrm{~cm}^{-1}$ to $2000 \mathrm{~cm}^{-1}$ (i.e., from $\approx 545$ to $\approx 595 \mathrm{~nm}$ ).

Morimoto et al. in 2020 presented a 1 Mpixel SPAD camera [71] characterized by a $3.8 \mathrm{~ns}$ time gating and a $24 \mathrm{kfps}$ frame rate. This sensor represents one of the most notable achievements regarding this research field. It was used to capture $2 \mathrm{D} / 3 \mathrm{D}$ scenes over $2 \mathrm{~m}$ with resolution of $5.4 \mathrm{~mm}$ and precision better than $7.8 \mathrm{~mm}(\mathrm{rms})$. The camera was composed by two sections of $1024 \times 500$ pixels characterized by two different kinds of pixel.

The SPAD camera presented in [71] showed high performances also in complex imaging applications. In [164] it was employed to capture an ultrafast phenomena, such as the propagation of a light pulse. In this paper, the authors demonstrated that a video can be obtained by using a frame interval of $36 \mathrm{ps}$.

\subsection{Interferometry: A Possible Resource}

Interferometry could be employed to overcome the possible problems originating by the use of a SPAD array. A Fourier transform Raman spectrometer (FT-Raman) demonstrated to be a suitable approach allowing one to obtain the fluorescence rejection in Raman spectra by employing a near infrared (NIR) source $(1064 \mathrm{~nm})$ [170]. Nevertheless, in this case several drawbacks should be considered: NIR detectors have a remarkable cost and the Raman cross-section (i.e., $\lambda^{-4}$ ) suggests choosing a short excitation wavelength.

A further solution can be implemented by introducing a Fabry-Pérot interferometer (FPI) before the SPAD, allowing one to scan a wavelength range by moving only one of the two mirrors. Here an ideal configuration is considered: a FPI composed by two parallel partially reflective mirrors and an orthogonal monochromatic plane wave [171]. Real FPI are affected by several deficiencies [172] with consequences on the achievable spectral resolution.

The ideal resolution is determined only by the reflectivity of the mirrors, and thus, by the Finesse characterizing the designed optical cavity. In Figure 18 the results regarding two opposite configurations are reported. It can be straightforwardly concluded that it is possible to resolve the chosen 17 spectral elements in the visible range by suitably selecting the value of $R$.

In order to estimate the optimal setup to enable one to distinguish the 17 spectral elements previously introduced, a resolution power (RP) was defined. This choice was necessary because the curves reported in Figure 18a,b do not share the same width. This new parameter, which allows for a qualitative approach to the definition of the resolution capabilities of the designed setup, was evaluated as follows:

1. For each value of $R$, the FWHM (i.e., $w_{i d t h}$ ) of the curves T vs. $\lambda$ was suitably estimated.

2. If the sum of these values was higher (lower) than the wavelength interval which was considered, their ratio was higher (lower) than 1 ; i.e., $R P=\frac{\sum_{i} w i d t h_{i}}{\lambda_{\max }-\lambda_{\min }}>1(<1)$.

The results of further simulations are reported in Table 5 . These can be used to estimate the necessary reflectivity $R$ to be chosen in order to design a FPI able to resolve the desired wavelengths in the visible range. Two configurations were considered: a spectral resolution of $20 \mathrm{~nm}(2 \mathrm{~nm})$ and 17 (165) spectral elements to be resolved. In the first case the threshold value $R=0.994$ represents a reflectivity achievable with low cost optical elements, while in 
the second one, unusually high-reflectivity mirrors could be employed and specific optical elements with $R>0.99994$ should be realized.

a)

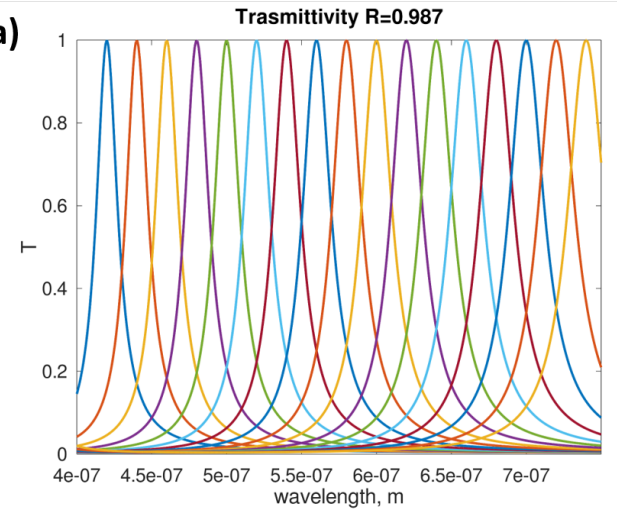

b)

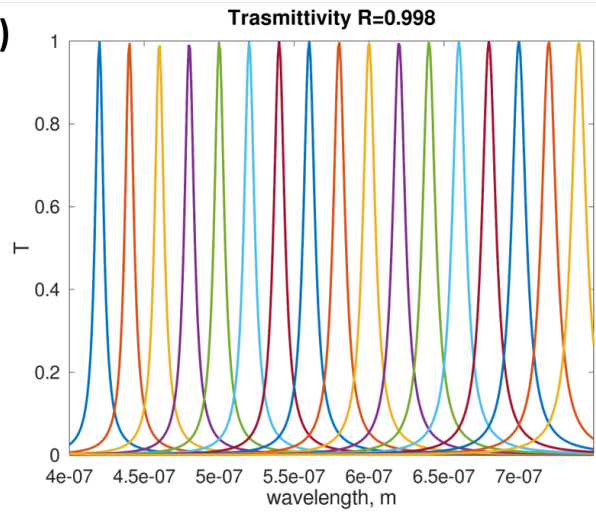

Figure 18. An ideal Fabry-Pérot interferometer (FPI) was considered; the first-order transmission maxima were obtained by scanning the distance $d$ from 210 to $350 \mathrm{~nm}$, with single steps equal to $10 \mathrm{~nm}$. (a) The transmission profile of an ideal FPI composed by two infinite and identical extent plates $(R=0.987)$, illuminated with a monochromatic plane wave at normal incidence; $(\mathbf{b})$ the same simulation was performed with $R=0.998$.

Furthermore, single steps of $10 \mathrm{~nm}$ can be realized with commercial solutions (e.g., piezo actuators) while smaller displacements can be affected by insufficient repeatability; it follows that this parameter assumes a fundamental role (to be carefully assessed) concerning the expected spectral resolution.

Table 5. Coefficient obtained with the polynomial fit resolution power (RP) vs. reflectivity $(R)$ : $y=a \cdot x^{2}+b \cdot x+c$.

\begin{tabular}{ccccc}
\hline Resolution [nm] & $\boldsymbol{a}$ & $\boldsymbol{b}$ & $\boldsymbol{c}$ & $\mathbf{R P}=\mathbf{1}$ \\
\hline 20 & -3002.6 & 5881.7 & -2878.7 & $R=0.994$ \\
2 & $-15,916,262.3$ & $31,822,936.8$ & $-15,906,674$ & $R=0.99994$ \\
\hline
\end{tabular}

\subsection{SPAD: Conclusions}

SPAD imagers have not reached widespread use for far, mainly being research prototypes or spin-off of devices designed in academic environments. Moreover, large arrays ( $>256$ channels) are not produced, so that for spectroscopic purposes they are not very competitive. Only small arrays can now be purchased (PhotonForce, a $32 \times 32$ time-resolved photon-counting camera [173]; MPD, with a $64 \times 32$ [174] camera). Other kinds of SPAD arrays on the market may either be derived from non-imaging silicon photo multipliers, used, for example, in clinical PET (Philips dSiPM); or from time-of-flight sensors (STMicroelectronics) [175]).

Beyond the experimental setups based on Kerr-gate, both PMTs [176,177] and ICCDs [178] have been employed in TGRS with sub-nanosecond gating. Though the latter kind of detectors are very sensitive, they are also much more expensive than SPADs; moreover, the electronic management of the devices is often embedded in the electronics and not completely customizable. The TG-SPAD-based CMOS pixel circuit can be used to reduce the cost and improve the integration of the detector. Nevertheless, it is worth noting that an array with few pixels, i.e., a few SPADs, could not guarantee the spectral resolution necessary for Raman spectroscopy. Moreover, due to the single-photon detection ability of the SPADs, it is not possible to acquire single-shot spectra, but accumulation of more laser shots is necessary, regardless of the single-pixel or array configuration. 


\section{Intensified CCD (ICCD)}

\subsection{Introduction and Theory}

Intensified ICCDs are CCDs coupled to devices called image intensifiers, which allow photon multiplication (i.e., image intensification). The image intensifier is usually composed by three different elements: a photocathode, a micro-channel plate (MCP) and a phosphor screen (see Figure 19).

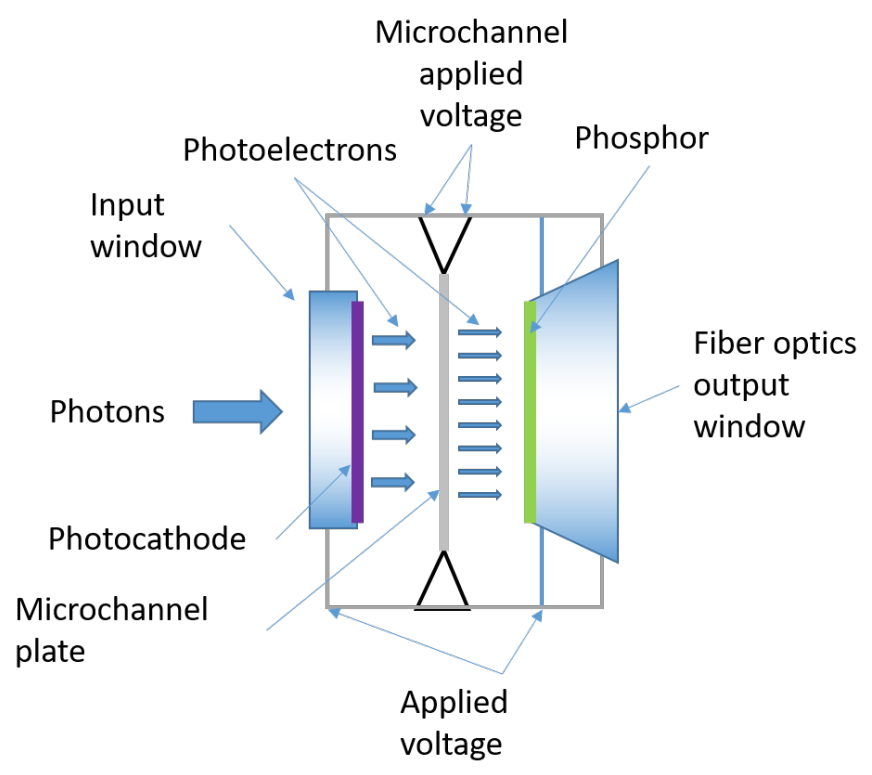

Figure 19. Intensified CCD (ICCD) scheme.

These elements are mounted in front of the CCD. Photons traveling towards the $\mathrm{CCD}$ hit the photocathode, generating photoelectrons. A control voltage, applied between the photocathode and MCP, accelerates these photoelectrons towards the MCP. This is composed by many thin glass capillaries (channels) whose internal walls are coated with a secondary electron emitting material. The electrons flying through the channels bump against the walls, and the repeated impacts cause them to multiply in number, so that the $\mathrm{MCP}$ acts as an electron multiplier device. The internal diameters of the channels range from 10 to $20 \mu \mathrm{m}$, bundled together to form a disk-shaped plate with a typical thickness of 0.5 to $1 \mathrm{~mm}$. Through this process, each single electron can be multiplied into as many as 104 at the exit of the MCP. These are thereafter accelerated towards the phosphor screen, which finally converts the multiplied electrons back to photons, guided to the CCD by a fiber optic or a lens. Thus, the number of photons collected by the CCD sensor is always much larger than the number of photons originating from the dark current noise. For this reason, it is less necessary to cool an ICCD camera with respect to other systems, which is described in the following. The ability to switch on and off the MCP control voltage allows an ICCD to gather light down to very short time lapses (Figure 20) and then reject part of the fluorescence signal.

Previous intensifiers, named Gen II, were equipped with different types of photocathode materials, which allowed one to customize the image intensifier to the spectral requirements of the specific application. They were based on bi- or multi-alkali photocathodes. Indeed, the spectral response of an ICCD camera is primarily determined by the photocathode material used in the image intensifier.

Photocathodes of Gen II intensifiers used to provide quantum efficiencies of the order of only $25 \%$. Gen III intensifiers, the third generation, also use the highly effective multi-channel plate, but in addition, they use semiconductor photocathodes (e.g., gallium arsenide photocathodes) that feature quantum efficiencies of more than $50 \%$. These materials are typically doped to "tune" the response to particular wavelength ranges. (See Table 6 for examples of photocathodes mounted on commercial Andor ICCD). 


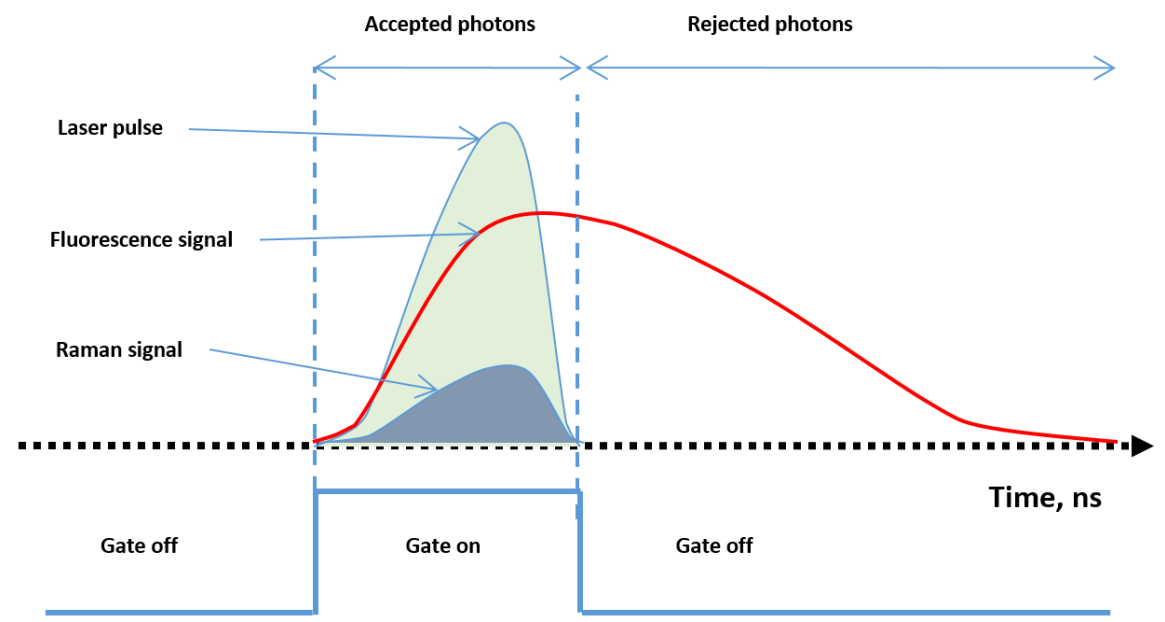

Figure 20. The time gating principle of ICCDs.

Table 6. Photocathodes mounted on the commercial Andor ICCD.

\begin{tabular}{cccc}
\hline Photocathode & Type & Peak QE & Min. Gating Speed \\
\hline 1 & Gen 2 & $18 \%$ & $<2 \mathrm{~ns}$ \\
2 & Gen 2 & $16 \%$ & $<5 \mathrm{~ns}$ \\
3 & Gen 2 & $13.5 \%$ & $<50 \mathrm{~ns}$ \\
4 & Gen 3 & $48 \%$ & $<2 \mathrm{~ns}$ \\
5 & Gen 3 & $26 \%$ & $<2 \mathrm{~ns}$ \\
6 & Gen 2 & $25 \%$ & $<100 \mathrm{~ns}$ \\
7 & Gen 3 & $4 \%$ & $<3 \mathrm{~ns}$ \\
8 & Gen 3 & $40 \%$ & $<2 \mathrm{~ns}$ \\
9 & Gen 2 & $22 \%$ & $<2 \mathrm{~ns}$ \\
\hline
\end{tabular}

The photocathodes can be applied to a quartz window that allows the photocathode response to extend into the UV range $(\approx 180 \mathrm{~nm})$. The quartz window can be substituted with a magnesium fluoride window to provide a response into the VUV $(\approx 120 \mathrm{~nm})$. If only a glass window is available, the spectral response is limited to wavelengths greater than the $350 \mathrm{~nm}$.

Different photocathodes and their thicknesses can be "tuned" to optimize the wavelength response in different regions.

The primary impact of the intensifier is raising the signal-to-noise ratio, by amplifying the signal impacting the photocathode. On the other hand, in fact, the dark noise depends on the integration time and may derive from the thermal excitation of the photocathode and the CCD. The CCD can be cooled to reduce the dark current, while the photocathode cannot and produces a contribution that is amplified in the MCP. However, this is generally not an issue when using short gate times.

A second important effect of the presence of the intensifier is that it can be operated as a shutter: the emitted photoelectrons are accelerated towards the MCP only when a correct control voltage between the photocathode and the MCP is applied. When this voltage is reversed, the electrons return to the photocathode and there is no electron multiplication, nor is light emitted from the image intensifier. In this case the ICCD acts as a close shutter. This process, allowing image intensification only during the application of the control voltage at the photocathode is called gating. For this reason, ICCDs are also usually referred to as gateable CCDs.

Thus, besides the extremely high sensitivity of ICCD cameras, which enable single photon detection, the gateability is one of the major advantages of the ICCD. The best performing ICCD cameras enable shutter times as short as 200 ps (see Table 7 for a comparative report regarding three commercial products: Andor [179,180], Stanford [181,182] and Princeton [183]). 
The ICCD can operate in the free running mode; in this case the camera is periodically gated by the built-in trigger source with different trigger frequencies and frame rates.

ICCDs are used in night vision devices and can be employed in various scientific applications, including time-resolved Raman spectroscopy [11,184]; depth analysis and profiling of multi-layer materials [178]; time-resolved fluorescence spectroscopy [185], quantum imaging [54]; fluorescence lifetime imaging [186]; and fluorescence detection and imaging [187].

An alternative to the ICCD is the electron-multiplying CCD (EMCCD), which contains an electron multiplying CCD sensor. In this case, the incoming photons are directly collected by the CCD sensor. The photo electrons generated by the sensor are then read out and afterwards multiplied electronically in a multi-stage gain register. For this reason, EMCCD cameras need extremely strong cooling (i.e., around $170 \mathrm{~K}$ ), because the electrons originating from the dark current noise are of the same order of magnitude as the signal electrons under low-light conditions and are amplified together with them to the same extent. This powerful cooling system, however, contributes to raising the costs of an EMCCD and may yield condensation problems. Furthermore, EMCCDs do not provide as a fast gating capability as ICCD cameras do.

Anyway, the cost of EMCCDs is lower than ICCDs and and the resolution is usually better, since there is no need for the intensifier and larger sensors can be realized. Thus, EMCCDs may replace ICCDs in many applications that do not require time gating. Several studies were published regarding the comparison of ICCD/EMCCD/CCD and the errors related to each system $[188,189]$.

A different method for achieving very high temporal resolution is based on the use of a streak camera [190,191]. A streak camera is a device in which:

1. Photons are converted into electrons by a photocathode.

2. Electrons are accelerated and deflected by a high speed sweep voltage.

3. Electrons are projected across a phosphor screen.

4. Electrons are converted back to photons by the phosphor screen.

The deflection system allows one to convert temporal variations of the entrance signal in spatial coordinate along the sweeping direction (Figure 21). If a time dependant 1D spectrum is projected in the streak camera, the output is a 2D image in which each line represents the spectrum at a different time. With such a system, temporal resolutions as short as $200 \mathrm{fs}$ can be achieved. However, for Raman spectroscopy typical resolutions are of the order of tens of picoseconds.

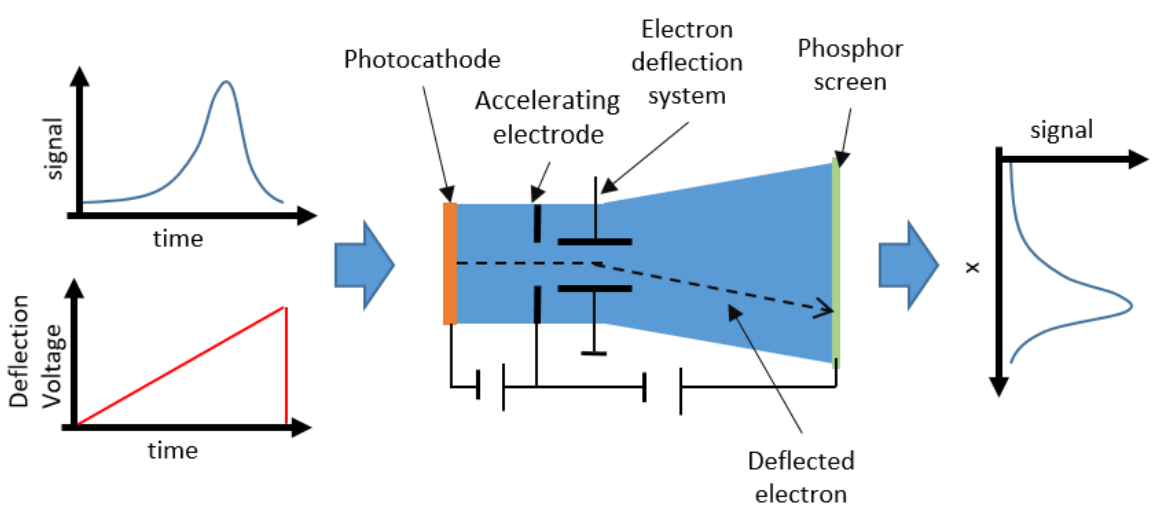

Figure 21. Working scheme of a streak camera. Through modulation of the electron deflection voltage, the deflection of the electrons emitted by the photocathode converts the time axis onto a spatial coordinate along the sweeping direction. If the entrance signal is a spectrum obtained by a dispersive medium, the streak camera allows one to resolve the intensity of the spectrum with time resolution as short as $200 \mathrm{fs}$. 
Table 7. Comparative analysis of the main commercial items: Andor [179,180], Stanford [181,182] and Princeton [183].

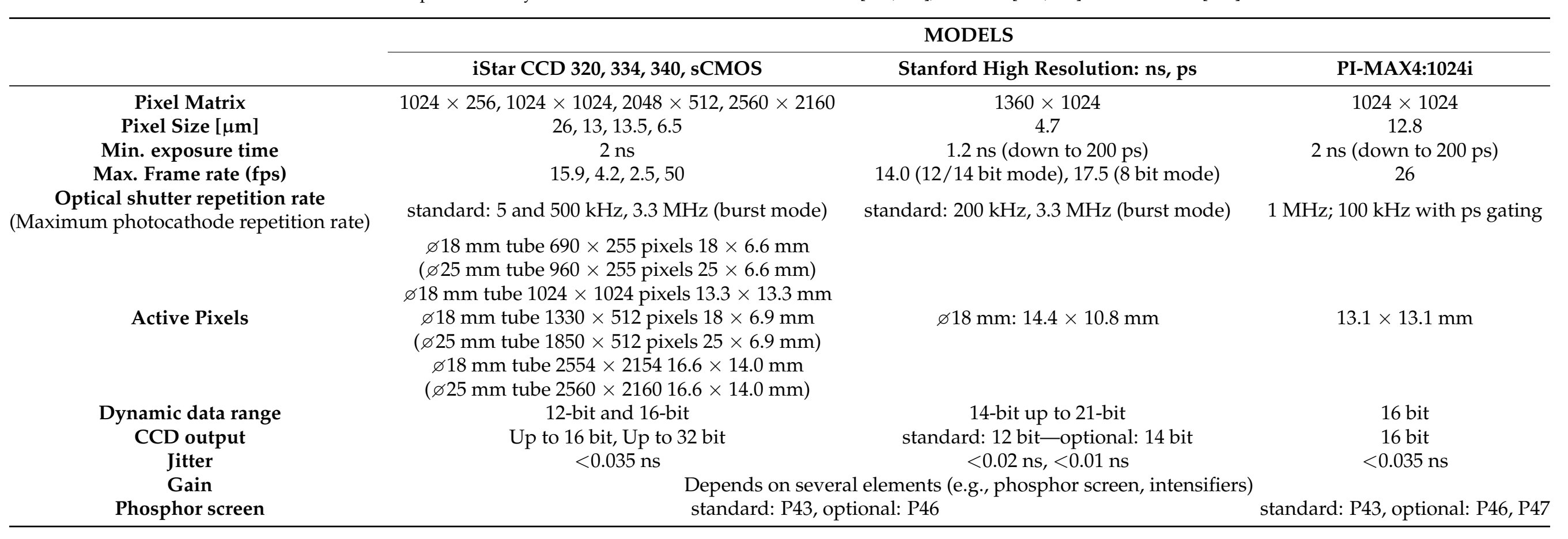




\subsection{State of the Art}

In [11] it was proven that a picosecond excitation pulse coupled to a sub-nanosecond gating can lead to effective rejection of most fluorescence signal. This experiment represents a development of a previous work dealing with ps Raman spectroscopy using a streak camera [192] (10 ps time resolution, up to $2 \mathrm{kHz}$ repetition rate) and a fluorophore with 4 ns of fluorescence lifetime.

It was possible to achieve a high level of background signal rejection both for solid and powder samples through a combination of an ordinary spectrograph coupled to an ultrafast, gated ICCD. A temporal resolution of about $150 \mathrm{ps}$ was achieved, after $785 \mathrm{~nm}$ Ti:Sa laser excitation (PRR: $76 \mathrm{MHz}$; pulse duration: 2 ps; average power: $300 \mathrm{~mW}$ ). It is worth noticing that the experiment was carried out without noticeable damage to the sample.

Since under a ps gating the number of Raman photons collected in one acquisition is usually very small, a high duty cycle was needed to obtain sufficient SNR. The image intensifier of the ICCD was able to work at very high repetition rate (up to $110 \mathrm{MHz}$ ) and very fast gating times (less than 200 ps, jitter lower than 20 ps). The photocathode employed was sensitive in the VIS-NIR (400-900 nm), and 65\% quantum efficiency, and had a final resolution of $1376 \times 1040$ pixels.

The suitability of this approach for rejection of fluorescence was demonstrated in resonance Raman spectroscopy (RRS) by different groups [193]. Resonance in Raman spectra [8] happens when the incident photon energy is near an electronic transition of the investigated molecule: then the probability of transition is greatly enhanced and the intensity of Raman scattering, making it possible to study chemical compounds at very low concentrations.

In [193] a Raman instrument was assembled and tested that rejects typically $98-99 \%$ of background fluorescence. Use is made of ps laser pulses and an ultrafast ICCD for the time-gated detection in order to record the Raman signals during the pulse while blocking most of the fluorescence. The gated intensifier can be operated at $80 \mathrm{MHz}$, so high repetition rates and low pulse energies can be used, thereby minimizing photodegradation. For excitation, they use a frequency-tripled or doubled Ti:sapphire laser with a pulse width of 3 ps. It cannot be shorter because of the necessary spectral resolution for the RRS. The total instrumental resolution was $7 \mathrm{~cm}^{-1}$ in the the blue region and $15 \mathrm{~cm}^{-1}$ in the ultraviolet (UV) region. This setup allows one to use RRS for extra sensitivity and selectivity, even in the case of strong background fluorescence, and excitation wavelengths in the visible or UV range could be employed.

In [178] it was demonstrated that effective fluorescence rejection can be achieved using a low peak power, high repetition rate Ti:sapphire laser coupled to a fast-gated ICCD. This technical resource represents a useful tool in order to implement depth analysis and profiling of multi-layer materials with high performance, even in turbid conditions. In clear media, a confocal microscope works reasonably well to obtain depth resolution, although Raman signals originating from points above or below the focal point, though defocused, are collected and affect the depth resolution. In turbid media, both the excitation and Raman photons undergo multiple scattering events in all directions, so the focusing of deeper layers and the achievement of high depth resolution are usually problematic.

This approach seems to be promising with respect to the other ones reported in literature [194].

Time-gated Raman detection in depth profiling can be reached through time-gated acquisition after an appropriate delay; this allows one to discriminate between Raman photons originating from different layers. A laser excitation at $398 \mathrm{~nm}$ delivered $3 \mathrm{ps}$ pulses at $76 \mathrm{MHz}$ PRR. Tests over 1 and $2 \mathrm{~mm}$ of white powders and solid white polymers, respectively, allowed the evaluation of temporal discrimination and depth selectivity of the system on different combinations of first and second layers.

In [184], ICCDs were employed for standoff detection and identification of explosives and hazardous chemicals using TRRS. This system can be useful when it is not possible to employ the conventional ones based on Raman spectroscopy in a backscattering 
configuration, while employing a compact CCD-coupled spectrograph and a CW laser. Although these hand-held detectors can be made quite portable, they can be affected by a relevant drawback, since the very low signal could be dominated by the high fluorescence background from substrate or the sample itself. This signal can be suppressed using ICCDs since the time gating of these detectors can be synchronized with the laser pulse to allow data collection only in a short time after the Raman emission.

After rejecting the spurious light, the Raman signal from the sample is intensified by the ICCD. A transportable TGRS system was developed to detect and identify traces of explosives or derivatives on surfaces $10 \mathrm{~m}$ away from the sensor, and was tested in a simulated real field scenario. The system exploits as an excitation source the second harmonic of a Nd:YAG laser (wavelength: $532 \mathrm{~nm}$; pulse duration: $4 \mathrm{~ns}$; pulse energy: $\approx 100 \mathrm{~mJ}$; PRR: $10 \mathrm{~Hz}$ ). The signal is collected by an $8^{\prime \prime}$ telescope in monostatic geometry and sent to a Czerny turner spectrograph coupled to a gated ICCD. This geometry facilitates the focusing of the system at different distances to allow standoff identification from the suspected samples in an easier way. A variable time delay, whose width is of the same order of laser pulse duration, was imposed from the laser Q-switch trigger to the gate according to the distance from the target (i.e., $6.6 \mathrm{~ns}$ for each meter of distance). Good rejection of background light and fluorescence was achieved using a gate width of $10 \mathrm{~ns}$ (similar to the laser pulse duration). This timing capability, however, does not allow a very effective rejection of the short-lived fluorophores (of the order of ns).

A further example of setup designed for the standoff Raman spectroscopy is reported in [195]; here the tests were performed even on common materials involved in narcotics manufacture, and cocaine and heroin.

\subsection{ICCD: Conclusions}

Compared to the other approaches presented and discussed (i.e., the Kerr-gate and SPADs, offering ps and tens of a ps time resolution, respectively), at the current time, state-of-the-art ICCDs cannot reach the same temporal discrimination capabilities (the fastest ICCD show $200 \mathrm{ps}$ as the shortest gating time). Nevertheless, for particular samples and applications (e.g., depth profiling or Raman analysis from standoff targets), the gated ICCDs shows good performances. On the other hand, for applications at the shortest distances, such as discrimination or selective probing of sub-millimetric layers, ICCDs are not fast enough, and setups involving both Kerr-gated or SPADs should be preferred. Moreover, the large dynamic range of CCDs allows single-shot collection of spectra, which is a key point in some applications, where the dose is limited by regulations or photodamage of the sample.

Alternatives to CCDs could come from CMOS sensors. Although a CMOS camera offers advantages in cost, integration possibilities, power dissipation and system size when compared to a CCD, the latter offers lower noise and better SNR for photon detection up to the near-infrared region [196].

\section{Summary and Conclusive Remarks}

Weak Raman signals often suffer from overimposed fluorescence, both from the substrate and from the sample itself. In Section 1 we discussed the many approaches adopted to reduce the contribution of the fluoresce signal, whose fluctuations may completely mask the weaker Raman signal, aiming to weakening the fluorescence or to enhance the Raman instead. In this work we gave an overview of the specific, but relevant, sub-fields of time domain gating for cutting of the fluorescence signal, based on the different lifetimes of the two phenomena.

A theoretical derivation of performances achievable by time gated techniques in terms of signal-to-noise ratio was presented in Section 2. In Section 3, the properties of optical Kerr cell gating devices were investigated, and a MonteCarlo simulation of possible reductions in the efficiency of a Kerr gate by fluctuations of operational parameters (laser energy, optical inhomogeneity of the nonlinear medium, etc.) was discussed. Many realizations 
of Kerr cell-based gating devices were reported. In Section 4 the working principle and applications of single photon avalanche diodes were described, and in Section 5 the same was done with CCD sensors, both intensified by a microchannel plate or coupled with a streak camera.

In the end, these different approaches for fast time gating spectroscopy have been presented in this paper, and it was discussed how they differ in terms of performances, advantages, drawbacks and costs, as summarized in the following:

- Features

1. Kerr-gate: the setup is based on a ps pulsed laser and a nonlinear medium acting as a switch when suitably activated. The detection should be implemented through a spectrometer coupled with a CCD or a CMOS.

2. ICCD: The gating is fully accomplished by using the camera intensifier.

3. TG-SPAD: The gating is realized by driving a SPAD with suitable electronics. Two different approaches can be followed: either a single SPAD spanning the wavelength range to be detected, or a SPAD array, measuring simultaneously all the wavelengths dispersed by a grating.

- Performances

1. Kerr-gate: ps time-domain Raman spectroscopy can be achieved. The gating window has a similar duration as the employed laser pulse.

2. ICCD: for particular samples and applications, the ICCD gate shows good performances. It is very sensitive and can improve the signal-to-noise ratio, allowing low-exposure spectroscopy.

3. TG-SPAD: the temporal resolution can go from tens to hundreds of picoseconds and is related even to the pulse width of the laser.

- Drawbacks

1. Kerr-gate: The gating and gated pulses must be accurately synchronized; the setup can reach remarkable size; high pulse energies are required; the activation time of the nonlinear medium can affect the theoretical performances achievable for a ps pulsed laser; the incomplete polarization rotation in the Kerr medium, the losses in optical elements and the optical transmittances of the Kerr gate do not allow one to obtain high efficiencies.

2. ICCD: The performances are limited by the technology implemented in this kind of item. The temporal discrimination power of current ICCDs can only reach hundreds of picoseconds, making its exploitation and application limited when faster processes have to be investigated.

3. TG-SPAD: Most SPAD imagers are still research prototypes, and only some characterized by limited size are commercially available.

- Cost

It is high for all of the methods, though significant differences should be considered. In any case, a ps laser is necessary and can be expensive. Often it represents the main issue to be faced.

1. Kerr-gate: the passive optical components that constitute the Kerr cell are relatively inexpensive.

2. ICCD: the cost of the ICCD can surpass the cost of the laser, likely becoming the most expensive setup in the comparison.

3. TG-SPAD: intermediate in cost compared to the previous solutions; it can be used to contain costs and improve the integration capability of the detection setup with the electronics of the developed device.

All the discussed methods can be used to extract the Raman signal when embedded with the fluorescence one, obtain a depth profiling or minimize the effect of photon scattering in thick samples and to reduce the statistical noise. 
However, in addition to the presented methods, frequency-domain methods [197-199], wavelength-domain methods [200-203] and computational methods [204-213] represent further approaches that could be implemented, although with lower performances [3].

Author Contributions: A.C. and F.A. contributed to the conceptualization, investigation, resources and visualization; writing-original draft preparation, A.C.; writing-review and editing, F.A.; supervision, F.A. All authors have read and agreed to the published version of the manuscript.

Funding: This research received no external funding.

Acknowledgments: The authors thank C. Ferrante and F. Colao for valuable discussions.

Conflicts of Interest: The authors declare no conflict of interest.

\section{References}

1. Rostron, P.; Gaber, S.; Gaber, D. Raman spectroscopy, review. Int. J. Eng. Res. 2016, 6, 50-64.

2. Lakowicz, J.R. Principles of Fluorescence Spectroscopy; Springer Science \& Business Media: New York, NY, USA, 2013.

3. Dong, W.; Chen, S.; Liu, Q. Review of fluorescence suppression techniques in Raman spectroscopy. Appl. Spectrosc. Rev. 2015, 55, 387-406.

4. McNay, G.; Eustace, D.; Smith, W.E.; Faulds, K.; Graham, D. Surface-enhanced Raman scattering (SERS) and surface-enhanced resonance Raman scattering (SERRS): A review of applications. Appl. Spectrosc. 2011, 65, 825-837. [CrossRef]

5. Pilot, R.; Signorini, R.; Durante, C.; Orian, L.; Bhamidipati, M.; Fabris, L. A review on surface-enhanced Raman scattering. Biosensors 2019, 9, 57. [CrossRef] [PubMed]

6. Tolles, W.M.; Nibler, J.W.; McDonald, J.R.; Harvey, A.B. A review of the theory and application of coherent anti-Stokes Raman spectroscopy (CARS). Appl. Spectrosc. 1977, 31, 253-271. [CrossRef]

7. Gebrekidan, M.T.; Knipfer, C.; Stelzle, F.; Popp, J.; Will, S.; Braeuer, A. A shifted-excitation Raman difference spectroscopy (SERDS) evaluation strategy for the efficient isolation of Raman spectra from extreme fluorescence interference. J. Raman Spectrosc. 2016, 47, 198-209. [CrossRef]

8. Strommen, D.P.; Nakamoto, K. Resonance raman spectroscopy. J. Chem. Educ. 1977, 54, 474. [CrossRef]

9. Angelini, F.; Colao, F. Optimization of laser wavelength, power and pulse duration for eye-safe Raman spectroscopy. J. Eur. Opt. Soc. Rapid Publ. 2019, 15, 2. [CrossRef]

10. Chadwick, C.T.; Willitsford, A.H.; Philbrick, C.R.; Hallen, H.D. Deep ultraviolet Raman spectroscopy: A resonance-absorption trade-off illustrated by diluted liquid benzene. J. Appl. Phys. 2015, 118, 243101. [CrossRef]

11. Martyshkin, D.V.; Ahuja, R.C.; Kudriavtsev, A.; Mirov, S.B. Effective suppression of fluorescence light in Raman measurements using ultrafast time gated charge coupled device camera. Rev. Sci. Instrum. 2004, 75, 630-635. [CrossRef]

12. Wohlmuth, M.; Pflaum, C.; Altmann, K.; Paster, M.; Hahn, C. Dynamic multimode analysis of Q-switched solid state laser cavities. Opt. Express 2009, 17, 17303-17316. [CrossRef] [PubMed]

13. Bizjak, A.; Nemes, K.; Mozina, J. Rotating-mirror Q-switched Er: YAG laser for optodynamic studies. J. Mech. Eng. 2011, 57, 3-10. [CrossRef]

14. Van Duyne, R.P.; Jeanmaire, D.L.; Shriver, D.F. Mode-locked laser Raman spectroscopy. New technique for the rejection of interfering background luminescence signals. Anal. Chem. 1974, 46, 213-222. [CrossRef]

15. Boyd, R.W. Nonlinear Optics, 2nd ed.; Academic Press: San Diego, CA, USA, 2003.

16. Saleh, B.E.A.; Teich, M.C. Fundamentals of Photonics, 1st ed.; Wiley: Hoboken, NJ, USA, 1991.

17. Matousek, P.; Towrie, M.; Ma, C.; Kwok, W.M.; Phillips, D.; Toner W.T.; Parker, A.W. Fluorescence suppression in resonance Raman spectroscopy using a high-performance ps Kerr gate. J. Raman Spectrosc. 2001, 32, 983-988. [CrossRef]

18. Knorr, F.; Smith, Z.J.; Wachsmann-Hogiu, S. Development of a time-gated system for Raman spectroscopy of biological samples. Opt. Express 2010, 18, 20049-20058. [CrossRef]

19. Yakovlev, V.V. Time gated confocal Raman microscopy: System design and its applications. In Proceedings of the SPIE, Imaging, Manipulation, and Analysis of Biomolecules, Cells, and Tissues V, San Jose, CA, USA, 20-25 January 2007; Volume 6441, p. 64411H.

20. Tan, W.; Yang, Y.; Si, J.; Tong, J.; Yi, W.; Chen, F.; Hou, X. Shape measurement of objects using an ultrafast optical Kerr gate of bismuth glass. J. Appl. Phys. 2010, 107, 043104.

21. Matousek, P.; Everall, N.; Towrie, M.; Parker, A.W. Depth Profiling in Diffusely Scattering Media Using Raman Spectroscopy and ps Kerr Gating. Appl. Spectrosc. 2005, 59, 200-205. [CrossRef] [PubMed]

22. Schmidt, B.; Laimgruber, S.; Zinth, W.; Gilch, P. A broadband Kerr shutter for femtosecond fluorescence spectroscopy. Appl. Phys. B 2003, 76, 809-814. [CrossRef]

23. Everall, N.; Hahn, T.; Matousek, P.; Parker, A.W.; Towrie, M. ps Time-Resolved Raman Spectroscopy of Solids: Capabilities and Limitations for Fluorescence Rejection and the Influence of Diffuse Reflectance. Appl. Spectrosc. 2001, 55, 1701-1708. [CrossRef]

24. Ganeev, R.A.; Ryasnyanski, A.I.; Kuroda, H. Nonlinear Optical Characteristics of Carbon Disulfide. Opt. Spectrosc. 2006, 100, 108-118. [CrossRef] 
25. Kim, M.J.; Hwang, J.; Wu, J.W. ps Nonlinear Optical Transmission Measurement on Anatase $\mathrm{TiO}_{2}$ Thin Films. J. Korean Phys. Soc. 2006, 49, 938-942.

26. Dimitrov, V.; Sakka, S. Linear and nonlinear optical properties of simple oxides. II. J. Appl. Phys. 1996, 79, 1741-1745. [CrossRef]

27. Vissers, E.J.P. Optical Nonlinearity in Titanium Dioxide Waveguides. Master's Thesis, University of Twente, Enschede, The Netherlands, 2018.

28. Yang, T.; Park, S.J.; Kim, T.G.; Shin, D.S.; Suh, K.D.; Park, J. Ultraviolet photodetector using pn junction formed by transferrable hollow n- $\mathrm{TiO}_{2}$ nanospheres monolayer. Appl. Spectrosc. 2017, 25, 30843-30850.

29. Lin, C.P.; Chen, H.; Nakaruk, A.; Koshy, P.; Sorrell, C.C. Effect of Annealing Temperature on the Photocatalytic Activity of TiO 2 Thin Films. Energy Procedia 2013, 34, 627-636. [CrossRef]

30. Wojcieszak, D.; Mazur, M.; Kurnatowska, M.; Kaczmarek, D.; Domaradzki, J.; Kepinski, L.; Chojnacki, K. Influence of Nd-Doping on Photocatalytic Properties of $\mathrm{TiO}_{2}$ Nanoparticles and Thin Film Coatings. Int. J. Photoenergy 2014, 2014, 463034. [CrossRef]

31. Kinoshita, S.; Ozawa, H.; Kanematsu, Y.; Tanaka, Y.; Sugimoto, N.; Fujiwara, S. Efficient optical Kerr shutter for femtosecond time-resolved luminescence spectroscopy. Rev. Sci. Instrum. 2000, 71, 3317. [CrossRef]

32. Fowles, G.R. Matrix Representation of Polarization. The Jones Calculus. In Introduction to Modern Optics, 2nd ed.; Dover Publications Inc.: New York, NY, USA, 1989.

33. Thakur, S.; Semnani, B.; Safavi-Naeini, S.; Majedi, A.H. Experimental Characterization of the Ultrafast, Tunable and Broadband Optical Kerr Nonlinearity in Graphene. Sci. Rep. 2019, 9, 10540. [CrossRef]

34. Aber, J.E.; Newstein, M.C.; Garetz, B.A. Femtosecond optical Kerr effect measurements in silicate glasses. JOSA B 2000, 17, 120-127. [CrossRef]

35. Hoffmann, M.C.; Brandt, N.C.; Hwang, H.Y.; Yeh, K.L.; Nelson, K.A. Terahertz Kerr effect. Appl. Phys. Lett. 2009, 95, 231105. [CrossRef]

36. Pontecorvo, E.; Ferrante, C.; Elles, C.G.; Scopigno, T. Spectrally tailored narrowband pulses for femtosecond stimulated Raman spectroscopy in the range 330-750 nm. Opt. Express 2013, 21, 6866-6872. [CrossRef] [PubMed]

37. Batignani, G.; Ferrante, C.; Fumero, G.; Scopigno, T. Broadband Impulsive Stimulated Raman Scattering Based on a Chirped Detection. J. Phys. Chem. Lett. 2019, 10, 7789-7796. [CrossRef]

38. Eksma Beam Shaper. Available online: http://eksmaoptics.com/out/media/EKSMA_Optics_Top_Hat_Beam_Shaping_Lenses_ FBS.pdf (accessed on 9 March 2021).

39. Matousek, P.; Towrie, M.; Stanley, A.; Parker, A.W. Efficient rejection of fluorescence from Raman spectra using ps Kerr gating. Appl. Spectrosc. 1999, 53, 1485-1489. [CrossRef]

40. El-Desouki, M.M.; Palubiak, D.; Deen, M.; Fang Q.; Marinov, O. A novel, high-dynamic-range, high-speed, and high-sensitivity CMOS imager using time-domain single-photon counting and avalanche photodiodes. IEEE Sens. J. 2011, 11, 1078-1083. [CrossRef]

41. El-Desouki, M.M.; Marinov, O.; Deen, M.; Fang, Q. CMOS active-pixel sensor with in-situ memory for ultrahigh-speed imaging. IEEE Sens. J. 2011, 11, 1375-1379. [CrossRef]

42. Palubiak D.P.; Deen, M. CMOS SPADs: Design issues and research challenges for detectors, circuits, and arrays. IEEE J. Sel. Top. Quantum Electron. 2014, 20, 409-426. [CrossRef]

43. Li, D.; Arlt, J.; Richardson, J.; Walker, R.; Buts, A.; Stoppa, D.; Charbon E.; Henderson, R. Real-time fluorescence lifetime imaging system with a $32 \times 320.13 \mu \mathrm{m}$ CMOS low dark-count single-photon avalanche diode array. Opt. Express 2010, 18, 10257-10269. [CrossRef]

44. Pancheri, L.; Stoppa, D. A SPAD-based pixel linear array for high-speed time-gated fluorescence lifetime imaging. In Proceedings of the ESSCIRC, Athens, Greece, 14-18 September 2009; Volume 8, pp. 428-431.

45. Castello, M.; Tortarolo, G.; Buttafavai, R.; Deguchi, T.; Villa, F.; Koho, S.; Pesce, L.; Oneto, M.; Pelicci, S.; Lanzanó, L.; et al. A robust and versatile platform for image scanning microscopy enabling super-resolution FLIM. Nat. Methods 2019, 16, 175-178. [CrossRef] [PubMed]

46. Slenders, E.; Castello, M.; Buttafava, M.; Villa, F.; Tosi, A.; Lanzanó, L.; Valtteri Koho, S.; Vicidomini, G. Confocal-based fluorescence fluctuation spectroscopy with a SPAD array detector. Light Sci. Appl. 2021, 10, 31. [CrossRef]

47. Tosi, A.; Mora, A.D.; Zappa, F.; Gulinatti, A.; Contini, D.; Pifferi, A.; Spinelli, L.; Torricelli, A.; Cubeddu, R. Fast-gated singlephoton counting technique widens dynamic range and speeds up acquisition time in time-resolved measurements. Opt. Express 2011, 19, 10735-10746. [CrossRef]

48. Dalla Mora, A.; Tosi, A.; Zappa, F.; Cova, S.; Contini, D.; Pifferi, A.; Spinelli, L.; Torricelli A.; Cubeddu, R. Fast-gated single-photon avalanche diode for wide dynamic range near infrared spectroscopy. IEEE J. Sel. Top. Quantum Electron. 2010, 16, 1023-1030 [CrossRef]

49. Niclass, C.; Soga, M.; Matsubara, H.; Ogawa M.; Kagami, M. A $0.18 \mu \mathrm{m}$ CMOS SoC for a 100 m-range 10 fps $200 \times 96-$ ixel time-of-flight depth sensor. In Proceedings of the IEEE Solid State Circuits Conference, San Francisco, CA, USA, 17-21 February 2013; Volume 19, pp. 488-489.

50. Zhang, C.; Lindner, S.; Antolovic, I.; Wolf, M.; Charbon, E. A CMOS SPAD imager with collision detection and 128 dynamically reallocating TDCs for single-photon counting and 3D time-of-flight imaging. Sensors 2018, 18, 4016. [CrossRef] 
51. Villa, F.; Lussana, R.; Bronzi, D.; Tisa, S.; Tosi, A.; Zappa, F.; Dalla Mora A.; Contini, D.; Durini, D.; Weyers, S.; et al. CMOS Imager With 1024 SPADs and TDCs for Single-Photon Timing and 3-D Time-of-Flight. IEEE J. Sel. Top. Quantum Electron. 2014, 20, 364-373. [CrossRef]

52. Avella, A.; Piacentini, F.; Borsarelli, M.; Barbieri, M.; Gramegna, M.; Lussana, R.; Villa, F.; Tosi, A.; Degiovanni, I.P.; Genovese, M. Anomalous Weak Values and the Violation of a Multiple-measurement Leggett-Garg Inequality. Phys. Rev. A 2017, 96, 052123. [CrossRef]

53. Zhang, J.; Itzler, M.; Zbinden, H.; Pan, J.-W. Advances in InGaAs/InP single-photon detector systems for quantum communication. Light Sci. Appl. 2015, 4, e286. [CrossRef]

54. Aspden, R.S.; Gemmell, N.R.; Morris, P.A.; Tasca, D.S.; Mertens, L.; Tanner, M.G.; Kirkwood, R.A.; Ruggeri, A.; Tosi, A.; Boyd, R.W.; et al. Photon-sparse microscopy: Visible light imaging using infrared illumination. Optica 2015, 2, 1049-1052. [CrossRef]

55. Genovese, M. Photon-sparse microscopy: Visible light imaging using infrared illumination. J. Opt. 2016, 18, 073002. [CrossRef]

56. Maruyama, Y.; Blacksberg, J.; Charbon, E. A $1024 \times 8700$ ps timegated SPAD line sensor for planetary surface exploration with laser Raman spectroscopy and LIBS. IEEE J. Solid State Circuits 2014, 49, 179-189. [CrossRef]

57. Kostamovaara, J.; Tenhunen, J.; Kögler, M.; Nissinen, I.; Nissinen, J.; Keränen, P. Fluorescence suppression in Raman spectroscopy using a time-gated CMOS SPAD. Opt. Express 2013, 21, 31632-31645. [CrossRef] [PubMed]

58. Nissinen, I.; Nissinen, J.; Lansman, A.; Hallman, L.; Kilpela, A.; Kostamovaara, J.; Kogler, M.; Aikio, M.; Tenhunen, J. A sub-ns time-gated CMOS single photon avalanche diode detector for Raman spectroscopy. In Proceedings of the European Solid-State Device Research Conference, Helsinki, Finland, 12-16 September 2011; pp. 375-378.

59. Li, Z.; Deen, M.J. Towards a portable raman spectrometer using a concave grating and a time-gated CMOS SPAD. Opt. Express 2014, 22, 18736-18747. [CrossRef] [PubMed]

60. Charbon, E. Single-photon imaging in complementary metal oxide semiconductor processes. Philos. Trans. R. Soc. A 2014, 372, 20130100. [CrossRef]

61. Holma, J.; Nissinen, I.; Nissinen, J.; Kostamovaara, J. Characterization of the timing homogeneity in a CMOS SPAD array designed for time-gated Raman spectroscopy. IEEE Trans. Instrum. Meas. 2017, 66, 1837-1844. [CrossRef]

62. Nissinen, I.; Nissinen, J.; Keränen, P.; Stoppa, D.; Kostamovaara, J. A 16 × 256 SPAD line detector with a 50-ps, 3-bit, 256-channel time-to-digital converter for Raman spectroscopy. IEEE Sens. J. 2018, 18, 3789-3798. [CrossRef]

63. Kekkonen, J.; Talala, T.; Nissinen, J.; Nissinen, I. On the Spectral Quality of Time-Resolved CMOS SPAD-Based Raman Spectroscopy With High Fluorescence Backgrounds. IEEE Sens. J. 2020, 20, 4635-4645. [CrossRef]

64. Li, Z. Miniaturization of Time-Gated Raman Spectrometer with a Concave Grating and a CMOS Single Photon Avalanche Diode. Ph.D. Thesis, McMaster University, Hamilton, ON, Canada, 2015.

65. Gallivanoni, A.; Rech, I.; Ghioni, M. Progress in quenching circuits for single photon avalanche diodes. IEEE Trans. Nucl. Sci. 2010, 57, 3815-3826. [CrossRef]

66. Eisele, A.; Henderson, R.; Schmidtke, B.; Funk, T.; Grant, L.; Richardson, J.; Freude, W. 185 MHz count rate, 139 dB dynamic range single-photon avalanche diode with active quenching circuit in $130 \mathrm{~nm}$ CMOS technology. In Proceedings of the International Image Sensor Workshop, Hokkaido, Japan, 8-11 June 2011.

67. Palubiak, D.; El-Desouki, M.M.; Marinov, O.; Deen M.; Fang, Q. High-speed, single-photon avalanche-photodiode imager for biomedical applications. IEEE Sens. J. 2011, 11, 2401-2412. [CrossRef]

68. Bruschini, C.; Homulle, H.; Antolovic, I.M.; Burri S.; Charbon, E. Single-photon avalanche diode imagers in biophotonics: Review and outlook. Light Sci. Appl. 2019, 8, 87. [CrossRef] [PubMed]

69. Maruyama, Y.; Blacksberg, J.; Charbon, E. A time-resolved 128x128 SPAD camera for laser Raman spectroscopy. Proc. SPIE 2012, $8374,83740 \mathrm{~N}$.

70. Vilella, E.; Alonso, O.; Montiel, A.; Vilá, A.; Dieguez, A. A low-noise time-gated single-photon detector in a HV-CMOS technology for triggered imaging. Sens. Actuators A 2013, 201, 342-351. [CrossRef]

71. Morimoto,K.; Ardelean, A.; Wu, M.L.; Ulku, A.C.; Antolovic, I.M.; Bruschini, C.; Charbon, E. Megapixel time-gated SPAD image sensor for 2D and 3D imaging applications. Optica 2020, 7, 346-354. [CrossRef]

72. Rochas,A.; Gosch, M.; Serov, A.; Besse, P.A.; Popovic, R.S.; Lasser, T.; Rigler, R. First fully integrated 2-D array of single-photon detectors in standard CMOS technology. IEEE Photonics Technol. Lett. 2003, 15, 963-965. [CrossRef]

73. Michalet, X.; Ingargiola, A.; Colyer, R.A.; Scalia, G.; Weiss, S.; Maccagnani, P.; Gulinatti, A.; Rech, I.; Ghioni, M. Silicon photoncounting avalanche diodes for singlemolecule fluorescence spectroscopy. IEEE J. Sel. Top. Quantum Electron. 2014, 20, $248-267$. [CrossRef]

74. Rech, I.; Resnati, D.; Marangoni, S.; Ghioni, M.; Cova, S. Compact eight channel photon counting module with monolithic array detector. Proc. SPIE 2007, 6771, 677113.

75. Rech, I.; Marangoni, S.; Resnati, D.; Ghioni, M.; Cova, S. Multipixel single photon avalanche diode array for parallel photon counting applications. J. Mod. Opt. 2009, 56, 326-333. [CrossRef]

76. Michalet, X.; Colyer, R.A.; Scalia, G.; Kim, T.; Levi, M.; Aharoni, D.; Cheng, A.; Guerrieri, F.; Arisaka, K.; Millaud, J.; et al. High-throughput single-molecule fluorescence spectroscopy using parallel detection. Proc. SPIE 2010, 7608, 76082D. 
77. Michalet, X.; Colyer, R.A.; Scalia, G.; Ingargiola, A.; Lin, R.; Millaud, J.E.; Weiss, S.; Siegmund, O.H.W.; Tremsin, A.S.; Vallerga, J.V.; et al. Development of new photon-counting detectors for singlemolecule fluorescence microscopy. Philos. Trans. R. Soc. B 2013, 368, 20120035. [CrossRef]

78. Ingargiola, A.; Colyer, R.A.; Kim, D.; Panzeri, F.; Lin, R.; Gulinatti, A.; Rech, I.; Ghioni, M.; Weiss, S.; Michalet, X. Parallel multispot smFRET analysis using an 8 pixel SPAD array. Proc. SPIE 2012, 8228, 82280B.

79. Ingargiola, A.; Panzeri, F.; Sarkosh, N.; Gulinatti, A.; Rech, I.; Ghioni, M.; Weiss, S.; Michalet, X. 8-spot smFRET analysis using two 8 pixel SPAD arrays. Proc. SPIE 2013, 8590, 85900E.

80. Schwartz, D.E.; Charbon, E.; Shepard, K.L. A single-photon avalanche diode imager for fluorescence lifetime applications. In Proceedings of the IEEE Symposium in VLSI Circuits, Kyoto, Japan, 14-16 June 2007; pp. 144-145.

81. Schwartz, D.E.; Charbon, E.; Shepard, K.L. A single-photon avalanche diode array for fluorescence lifetime imaging microscopy. IEEE J. Solid State Circuits 2008, 43, 2546-2557. [CrossRef]

82. Niclass, C.; Favi, C.; Kluter, T.; Gersbach, M.; Charbon, E. $128 \times 128$ single photon image sensor with column-level 10-bit time-to-digital converter array. IEEE J. Solid State Circuits 2008, 43, 2977-2989. [CrossRef]

83. Pavia, J.M.; Wolf, M.; Charbon, E. Measurement and modeling of microlenses fabricated on single-photon avalanche diode arrays for fill factor recovery. Opt. Express 2014, 22, 4202-4213. [CrossRef]

84. Pavia, J.M.; Wolf, M.; Charbon, E. Single-photon avalanche diode imagers applied to near-infrared imaging. IEEE J. Sel. Top. Quantum Electron. 2014, 20, 291-298. [CrossRef]

85. Boiko, D.; Gunther, N.J.; Brauer, N.; Sergio, M.; Niclass, C.; Beretta G.B.; Charbon, E. A quantum imager for intensity correlated photons. New J. Phys. 2009, 11, 013001. [CrossRef]

86. Boiko, D.; Gunther, N.J.; Brauer, N.; Sergio, M.; Niclass, C.; Beretta, G.B.; Charbon, E. On the application of a monolithic array for detecting intensity-correlated photons emitted by different source types. Opt. Express 2009, 17, 15087-15103. [CrossRef]

87. Homulle, H.; Powolny, F.; Stegehuis, P.L.; Dijkstra, J.; Li, D.U.; Homicsko, K.; Rimoldi, D.; Muehlethaler, K.; Prior, J.O.; Sinisi, R.; et al. Compact solid-state CMOS single-photon detector array for in vivo NIR fluorescence lifetime oncology measurements. Biomed. Opt. Express 2016, 7, 1797-1814. [CrossRef] [PubMed]

88. Niclass, C.; Favi, C.; Kluter, T.; Monnier, F.; Charbon, E. Single-photon synchronous detection. IEEE J. Solid State Circuits 2009, 44,1977-1989. [CrossRef]

89. Stegehuis, P.L.; Boonstra, M.C.; de Rooij, K.E.; Powolny, F.E.; Sinisi, R.; Homulle, H.; Bruschini, C.; Charbon, E.; van de Velde, C.J.H.; Lelieveldt, B.P.F.; et al. Fluorescence lifetime imaging to differentiate bound from unbound ICG-cRGD both in vitro; in vivo. Proc. SPIE 2015, 9313, 931300.

90. Vitali, M.; Bronzi, D.; Krmpot, A.J.; Nikolic, S.N.; Schmitt, F.J.; Junghans, C.; Tisa, S.; Friedrich Vukojevic, T.V.; Terenius, L.; Zappa, F.; et al. A single-photon avalanche camera for fluorescence lifetime imaging microscopy and correlation spectroscopy. IEEE J. Sel. Top. Quantum Electron. 2014, 20, 344-353. [CrossRef]

91. Colyer, R.A.; Scalia, G.; Villa, F.; Guerrieri, F.; Tisa, S.; Zappa, F.; Cova, S.; Weiss, S.; Michalet, X. Ultra high-throughput single molecule spectroscopy with a 1024 pixel SPAD. Proc. SPIE 2011, 7905, 790503.

92. Guerrieri, F.; Tisa, S.; Tosi, A.; Zappa, F. Fast single-photon imager acquires 1024 pixels at 100 kframe/s. Proc. SPIE 2009, $7249,72490 \mathrm{U}$.

93. Guerrieri, F.; Tisa, S.; Tosi, A.; Zappa, F. Two-dimensional SPAD imaging camera for photon counting. IEEE Photonics J. 2010, 2, 759-774. [CrossRef]

94. Gersbach, M.; Maruyama, Y.; Trimananda, R.; Fishburn, M.W.; Stoppa, D.; Richardson, J.A.; Walker, R.; Henderson, R.; Charbon, E. A time-resolved, low-noise single-photon image sensor fabricated in deep-submicron CMOS technology. IEEE J. Solid State Circuits 2012, 47, 1394-1407. [CrossRef]

95. Li, D.U.; Tyndall, D.; Walker, R.; Richardson, J.A.; Henderson, R.K.; Arlt, J.; Stoppa, D.; Charbon, E. Video-rate fluorescence lifetime imaging camera with CMOS single-photon avalanche diode arrays and high-speed imaging algorithm. J. Biomed. Opt 2011, 16, 096012. [CrossRef] [PubMed]

96. Richardson, J.; Walker, R.; Grant, L.; Stoppa, D.; Borghetti, F.; Charbon, E.; Gersbach, M.; Henderson, R.K. A $32 \times 3250$ ps resolution 10-bit time to digital converter array in $130 \mathrm{~nm}$ CMOS for time correlated imaging. In Proceedings of the IEEE Custom Integrated Circuits Conference, San Jose, CA, USA, 13-16 September 2009; pp. 77-80.

97. Krstajić, N.; Poland, S.; Levitt, J.; Walker, R.; Erdogan, A.; Ameer-Beg, S.; Henderson, R.K. 0.5 billion events per second time correlated single photon counting using CMOS SPAD arrays. Opt. Lett. 2015, 40, 4305-4308. [CrossRef] [PubMed]

98. Gersbach, M.; Trimananda, R.; Maruyama, Y.; Fishburn, M.; Stoppa, D.; Richardson, J.; Walker, R.; Henderson, R.K.; Charbon, E. High frame-rate TCSPC-FLIM using a novel SPAD-based image sensor. Proc. SPIE 2010, 7780, 77801H.

99. Li, D.U.; Ameer-Beg, S.; Arlt, J.; Tyndall, D.; Walker, R.; Matthews, D.R.; Visitkul, V.; Richardson, J.; Henderson, R.K. Time-domain fluorescence lifetime imaging techniques suitable for solid-state imaging sensor arrays. Sensors 2012, 12, 5650-5669. [CrossRef] [PubMed]

100. Poland, S.P.; Coelho, S.; Krstajić, N.; Tyndall, D.; Walker, R.J.; Monypenny, J.; Li, D.D.U.; Henderson, R.K.; Ameer-Beg, S.M. Development of a fast TCSPC FLIM-FRET imaging system. Proc. SPIE 2013, 8588, 85880X.

101. Poland, S.P.; Krstajić, N.; Coelho, S.; Tyndall, D.; Walker, R.J.; Devauges, V.; Morton, P.E.; Nicholas, N.S.; Richardson, J.; Li, D.D.U.; et al. Time-resolved multifocal multiphoton microscope for high speed FRET imaging in vivo. Opt. Lett. 2014, 39, 6013-6016. [CrossRef] [PubMed] 
102. Poland, S.P.; Krstajić, N.; Monypenny, J.; Coelho, S.; Tyndall, D.; Walker, R.J.; Devauges, V.; Richardson, J.; Dutton, N.; Barber, P.; et al. A high speed multifocal multiphoton fluorescence lifetime imaging microscope for live-cell FRET imaging. Biomed. Opt. Express 2015, 6, 277-296. [CrossRef]

103. Poland, S.P.; Erdogan, A.T.; Krstajić, N.; Levitt, J.; Devauges, V.; Walker, R.J.; Li, D.U.; Ameer-Beg, S.M.; Henderson, R.K. New high-speed centre of mass method incorporating background subtraction for accurate determination of fluorescence lifetime. Opt. Express 2016, 24, 6899-6915. [CrossRef]

104. Kloster-Landsberg, M.; Tyndall, D.; Wang, I.; Walker, R.; Richardson, J.; Henderson, R.; Delon, A. Multi-confocal fluorescence correlation spectroscopy in living cells using a complementary metal oxide semiconductorsingle photon avalanche diode array. Rev. Sci. Instrum. 2013, 84, 076105. [CrossRef]

105. Singh, A.P.; Krieger, J.W.; Buchholz, J.; Charbon, E.; Langowski, J.; Wohland, T. The performance of 2D array detectors for light sheet based fluorescence correlation spectroscopy. Opt. Express 2013, 21, 8652-8668. [CrossRef]

106. Carrara, L.; Niclass, C.; Scheidegger, N.; Shea, H.; Charbon, E. A gamma, X-ray and high energy proton radiation-tolerant CIS for space applications. In Proceedings of the IEEE International Solid-State Circuits Conference, San Francisco, CA, USA, 8-12 February 2009; pp. 40-41.

107. Stuker, F.; Baltes, C.; Dikaiou, K.; Vats, D.; Carrara, L.; Charbon, E.; Ripoll, J.; Rudin, M. A novel hybrid imaging system for simultaneous fluorescence molecular tomography and magnetic resonance imaging. In Proceedings of the Biomedical Optics and 3-D Imaging (BTuD1), Miami, FL, USA, 11-14 April 2010.

108. Stuker, F.; Baltes, C.; Dikaiou, K.; Vats, D.; Carrara, L.; Charbon, E.; Ripoll, J.; Rudin, M. Hybrid small animal imaging system combining magnetic resonance imaging with fluorescence tomography using single photon avalanche diode detectors. IEEE Trans. Med. Imaging 2011, 30, 1265-1273. [CrossRef] [PubMed]

109. Stoppa, D.; Mosconi, D.; Pancheri, L.; Gonzo, L. Single-photon avalanche diode CMOS sensor for time-resolved fluorescence measurements. IEEE Sens. J. 2009, 9, 1084-1090. [CrossRef]

110. Maruyama, Y.; Charbon, E. An all-digital, time-gated $128 \times 128$ SPAD array for on-chip, filter-less fluorescence detection. In Proceedings of the IEEE Solid-State Sensors Actuators and Microsystems Conference, Beijing, China, 5-9 June 2011; pp. 1180-1183.

111. Blacksberg, J.; Maruyama, Y.; Charbon, E.; Rossman, G.R. Fast single-photon avalanche diode arrays for laser Raman spectroscopy. Opt. Lett. 2011, 36, 3672-3674. [CrossRef] [PubMed]

112. Veerappan, C.; Richardson, J.; Walker, R.; Li, D.U.; Fishburn, M.W.; Maruyama, Y.; Stoppa, D.; Borghetti, F.; Gersbach, M.; Henderson, R.K.; et al. A $160 \times 128$ single-photon image sensor with on-pixel 55 ps $10 \mathrm{~b}$ time-to-digital converter. In Proceedings of the 2011 IEEE International Solid-State Circuits Conference, San Francisco, CA, USA, 20-24 February 2011; Volume 16, p. 312314.

113. Veerappan, C.; Richardson, J.; Walker, R.; Li, D.U.; Fishburn, M.W.; Stoppa, D.; Borghetti, F.; Maruyama, Y.; Gersbach, M.; Henderson, R.K.; et al. Characterization of large-scale non-uniformities in a 20k TDC/SPAD array integrated in a $130 \mathrm{~nm}$ CMOS process. In Proceedings of the European Solid-State Device Research Conference, Helsinki, Finland, 12-16 September 2011; pp. 331-334.

114. Pancheri, L.; Massari, N.; Borghetti, F.; Stoppa, D. A $32 \times 32$ SPAD pixel array with nanosecond gating and analog readout. In Proceedings of the International Image Sensor Workshop, Hokkaido, Japan, 8-11 June 2011; pp. 1-4.

115. Pancheri, L.; Pasquardini, L.; Morganti, E.; Massari, N.; Stoppa, D.; Collini, C.; Lorenzelli, L.; Lunelli, L.; Pederzolli, C. Protein detection system based on $32 \times 32$ SPAD pixel array. Proc. SPIE 2012, 8439, 843913.

116. Pancheri, L.; Massari, N.; Stoppa, D. SPAD image sensor with analog counting pixel for time-resolved fluorescence detection. IEEE Trans. Electron Devices 2013, 60, 3442-3449. [CrossRef]

117. Durini, D.; Weyers, S.; Stühlmeyer, M.; Goehlich, A.; Brockherde, W.; Paschen, U.; Vogt, H.; Tisa, S.; Villa, F.; Bronzi, D.; et al. BackSPAD—Back-side illuminated single-photon avalanche diodes: Concept and preliminary performances. In Proceedings of the IEEE Symposium on Nuclear Science, Anaheim, CA, USA, 27 October-3 November 2012; pp. 1-2.

118. Zou, Y.; Bronzi, D. Villa, F.; Weyers, S. Backside Illuminated Wafer-to-Wafer Bonding Single Photon Avalanche Diode Array. In Proceedings of the Conference on Ph.D. Research in Microelectronics and Electronics (PRIME), Grenoble, France, 30 June-3 July 2014; pp. 1-4.

119. Arlt, J.; Tyndall, D.; Rae, B.R.; Li, D.D.U.; Richardson, J.A.; Henderson, R.K. A study of pile-up in integrated time-correlated single photon counting systems. Rev. Sci. Instrum. 2013, 84, 103105. [CrossRef]

120. Tyndall, D.; Rae, B.R.; Li, D.D.U.; Richardson, J.A.; Arlt, J.; Henderson, R.K. A 100 Mphoton/s time-resolved mini-silicon photomultiplier with on-chip fluorescence lifetime estimation in $0.13 \mu \mathrm{m}$ CMOS imaging technology. In Proceedings of the 2012 IEEE International Solid-State Circuits Conference, San Francisco, CA, USA, 19-23 February 2012; Volume 55, pp. $122-124$.

121. Tyndall, D.; Rae, B.R.; Li, D.D.U.; Arlt, J.; Johnston, A.; Richardson, J.A.; Henderson, R.K. A high-throughput time-resolved mini-silicon photomultiplier with embedded fluorescence lifetime estimation in $0.13 \mu \mathrm{m}$ CMOS. In Proceedings of the 2012 IEEE International Solid-State Circuits Conference, San Francisco, CA, USA, 19-23 February 2012; Volume 6, pp. 562-570.

122. Field, R.M.; Shepard, K.L. A 100 fps fluorescence lifetime imager in standard $0.13 \mu \mathrm{m}$ CMOS. In Proceedings of the IEEE Symposium on VLSI Circuits, Kyoto, Japan, 12-14 June 2013; pp. C10-C11.

123. Field, R.M.; Realov, S.; Shepard, K.L. A 100 fps, time-correlated singlephoton-counting-based fluorescence-lifetime imager in 130 nm CMOS. IEEE J. Solid State Circuits 2014, 49, 867-880. [CrossRef] 
124. Mandai, S.; Charbon, E. A $4 \times 4 \times 416$ digital SiPM array with 192 TDCs for multiple high-resolution timestamp acquisition. J. Instrum. 2013, 8, P05024. [CrossRef]

125. Maruyama, Y.; Blacksberg, J.; Charbon, E. A $1024 \times 8700$ ps time-gated SPAD line sensor for laser Raman spectroscopy and LIBS in space and roverbased planetary exploration. In Proceedings of IEEE International Solid-State Circuits Conference, San Francisco, CA, USA, 17-21 February 2013; pp. 110-111.

126. Nissinen, I.; Lansman, A.K.; Nissinen, J.; Holma, J.; Kostamovaara, J. A $2 \times(4 \times) 128$ time-gated CMOS single photon avalanche diode line detector with 100 ps resolution for Raman spectroscopy. In Proceedings of ESSCIRC, Bucharest, Romania, 16-20 September 2013; pp. 291-294.

127. Nissinen, I.; Nissinen, J.; P.Keränen Lansman, A.K.; Holma, J.; Kostamovaara, J. A $2 \times(4) \times 128$ multitime-gated SPAD line detector for pulsed Raman spectroscopy. IEEE Sens. J. 2015, 15, 1358-1365. [CrossRef]

128. Nissinen, I.; Nissinen, J.; Holma, J.; Kostamovaara, J. A $4 \times 128$ SPAD array with a 78 ps 512 channel TDC for time-gated pulsed Raman spectroscopy. Analog Integr. Circuits Signal Process 2015, 84, 353-362. [CrossRef]

129. Walker, R.J.; Braga, L.H.C.; Erdogan, A.T.; Gasparini, L.; Grant, L.A.; Henderson, R.K.; Massari, N.; Perenzoni, M.; Stoppa, D. A 92k SPAD time-resolved sensor in $0.13 \mu \mathrm{m}$ CIS technology for PET/MRI applications. In Proceedings of the International Image Sensor Workshop, Snowbird, UT, USA, 12-16 June 2013; pp. 1-4.

130. Braga, L.H.; Gasparini, L.; Grant, L.; Henderson, R.K.; Massari, N.; Perenzoni, M.; Stoppa, D.; Walker, R. A fully digital $8 \times 16$ SiPM array for PET applications with per-pixel TDCs and real-time energy output. IEEE J. Solid State Circuits 2014, 49, 301-314 [CrossRef]

131. Unternährer, M.; Bessire, B.; Gasparini, L.; Stoppa, D.; Stefanov, A. Coincidence detection of spatially correlated photon pairs with a monolithic timeresolving detector array. Opt. Express 2016, 24, 28829-28841. [CrossRef] [PubMed]

132. Gasparini, L.; Bessire, B.; Unternährer, M.; Stefanov, A.; Boikoc, D.; Perenzoni, M.; Stoppa, D. SUPERTWIN: Towards 100 kpixel CMOS quantum image sensors for quantum optics applications. In Proceedings of the SPIE Quantum Sensing and Nano Electronics Photonics XIV, San Francisco, CA, USA, 28 January-2 February 2017; Volume 10111, p. 101112L.

133. Burri, S.; Maruyama, Y.; Michalet, X.; Regazzoni, F.; Bruschini, C.; Charbon, E. Architecture and applications of a high resolution gated SPAD image sensor. Opt. Express 2014, 22, 17573-17589. [CrossRef] [PubMed]

134. Antolovic, I.M.; Burri, S.; Bruschini, C.; Hoebe, R.; Charbon, E. Nonuniformity analysis of a 65 kpixel CMOS SPAD imager. IEEE Trans. Electron Devices 2016, 63, 57-64. [CrossRef]

135. Wargocki, P.M.; Burri, S.; Bruschini, C.; Antolovic, I.M.; Charbon, E.; Goldys, E.M.; Spence, D.J. Imaging free and bound NADH towards cancer tissue detection using FLIM system based on SPAD array. In Proceedings of the European Conference on Lasers and Electro-Optics Europe and European Quantum Electronics Conference, Munich, Germany, 25-29 June 2017.

136. Antolovic, I.M.; Burri, S.; Bruschini, C.; Hoebe, R.A.; Charbon, E. Analyzing blinking effects in super resolution localization microscopy with singlephoton SPAD imagers. Proc. SPIE 2016, 9714, 971406.

137. Antolovic, I.M.; Burri, S.; Bruschini, C.; Hoebe, R.A.; Charbon, E. SPAD imagers for super resolution localization microscopy enable analysis of fast fluorophore blinking. Sci. Rep. 2017, 7, 44108. [CrossRef]

138. Antolovic, I.M.; Burri, S.; Hoebe, R.A.; Maruyama, Y.; Bruschini, C.; Charbon, E. Photon-Counting Arrays for Time-Resolved Imaging. Sensors 2016, 16, 1005. [CrossRef] [PubMed]

139. Carimatto, A.; Mandai, S.; Venialgo, E.; Gong, T.; Borghi, G.; Schaart, D.R.; Charbon, E. A 67,392-SPAD PVTB-compensated multi-channel digital SiPM with 432 column-parallel 48 ps 17b TDCs for endoscopic time-of-flight. In Prooceedings of the IEEE International Solid-State Circuits Conference, San Francisco, CA, USA, 22-26 February 2015; pp. 1-3.

140. Krstajić, N.; Levitt, J.; Poland, S.; Ameer-Beg, S.; Henderson, R. $256 \times 2$ SPAD line sensor for time resolved fluorescence spectroscopy. Opt. Express 2015, 23, 5653-5669. [CrossRef] [PubMed]

141. Ehrlich, K.; Kufcsák, A.; Krstajić, N.; Henderson, R.K.; Thomson, R.R.; Tanner, M.G. Fibre optic time-resolved spectroscopy using CMOS-SPAD arrays. Proc. SPIE 2017, 10058, 100580H.

142. Parmesan, L.; Dutton, N.A.W.; Calder, N.J.; Krstajić, N.; Holmes, A.J.; Grant, L.A.; Henderson, R.K. A $256 \times 256$ SPAD array with in-pixel time to amplitude conversion for fluorescence lifetime imaging microscopy. In Proceedings of the International Image Sensor Workshop, Vaals, The Netherlands, 8-1 June 2015.

143. Pavia, J.M. Near-Infrared Optical Tomography with Single-Photon Avalanche Diode Image Sensors. Ph.D. Thesis, EPFLUniversity of Lausanne, Lausanne, Switzerland, 2015.

144. Pavia, J.M.; Scandini, M.; Lindner, S.; Wolf, M.; Charbon, E. A $1 \times 400$ backside-illuminated SPAD sensor with 49.7 ps resolution, $30 \mathrm{pJ} /$ sample TDCs fabricated in 3D CMOS technology for near-infrared optical tomography. IEEE J. Solid State Circuits 2015, 50, 2406-2418. [CrossRef]

145. AlAbbas, T.; Dutton, N.A.W.; Almer, O.; Pellegrini, S.; Henrion Y.; Henderson, R.K. Backside illuminated SPAD image sensor with $7.83 \mu \mathrm{m}$ pitch in 3D-Stacked CMOS technology. In Proceedings of the IEEE International Electron Devices Meeting, San Francisco, CA, USA, 3-7 December 2016; pp. 8.1.1-8.1.4.

146. Lee, C.; Johnson, B.; Jung, T.; Molnar, A. A $72 \times 60$ angle-sensitive SPAD imaging array for lens-less FLIM. Sensors 2016, 16, 1422. [CrossRef] [PubMed]

147. Burri, S.; Bruschini, C.; Charbon, E. LinoSPAD: A compact linear SPAD camera system with 64 FPGA-based TDC modules for versatile 50 ps resolution timeresolved imaging. Instruments 2017, 1, 6.1-6.21. [CrossRef] 
148. Burri, S.; Homulle, H.; Bruschini, C.; Charbon, E. LinoSPAD: A time-resolved $256 \times 1$ CMOS SPAD line sensor system featuring 64 FPGA-based TDC channels running at up to 8.5 giga-events per second. Proc. SPIE 2016, 9899, 98990D.

149. Perenzoni, M.; Massari, N.; Perenzoni, D.; Gasparini, L.; Stoppa, D. A $160 \times 120$ pixel analog-counting single-photon imager with time-gating and self-referenced column-parallel A/D conversion for fluorescence lifetime imaging. IEEE J. Solid State Circuits 2016, 51, 155-167.

150. Dutton, N.A.W.; Gyongy, I.; Parmesan, L.; Gnecchi, S.; Calder, N.; Rae, B.R.; Pellegrini, S.; Grant, L.A.; Henderson, R.K. A SPADbased QVGA image sensor for single-photon counting and quanta imaging. IEEE Trans. Electron Devices 2016, 63, 189-196. [CrossRef]

151. Gyongy, I.; Davies, A.; Gallinet, B.; Dutton, N.A.W.; Duncan, R.R.; Rickman, C.; Henderson, R.K.; Dalgarno P.A. Cylindrical microlensing for enhanced collection efficiency of small pixel SPAD arrays in single-molecule localisation microscopy. Opt. Express 2018, 26, 2280-2291. [CrossRef]

152. Gyongy, I.; Davies, A.; Dutton, N.A.W.; Duncan, R.R.; Rickman, C.; Henderson, R.K.; Dalgarno P.A. Smart-aggregation imaging for single molecule localisation with SPAD cameras. Sci. Rep. 2016, 6, 37349. [CrossRef]

153. Erdogan, A.T.; Walker, R.; Finlayson, N.; Krstajić, N.; Williams, G.O.S.; Henderson, R.K. A 16.5 giga events/s $1024 \times 8$ SPAD line sensor with per pixel zoomable 50 ps-6.4 ns/bin histogramming TDC. In Proceedings of the IEEE Symposium in VLSI Circuits, Kyoto, Japan, 5-8 June 2017; pp. C292-C293.

154. Kufcsák, A.; Erdogan, A.; Walker, R.; Ehrlich, K.; Tanner, M.; Megia-Fernandez, A.; Scholefield, E.; Emanuel, P.; Dhaliwal, K.; Bradley, M.; et al. Time-resolved spectroscopy at 19,000 lines per second using a CMOS SPAD line array enables advanced biophotonics applications. Opt. Express 2017, 25, 11103-11123. [CrossRef]

155. Lindner, S.; Zhang, C.; Antolovic, I.M.; Kalyanov, A.; Jiang, J.; Ahnen, L.; di Costanzo, A.; Pavia, J.M.; Majos, S.S.; Charbon, E.; et al. A novel $32 \times 32,224$ Mevents/s time resolved SPAD image sensor for near-infrared optical tomography. In Proceedings of the Biophotonics Congress: Biomedical Optics Congress (JTh5A.6), Hollywood, FL, USA, 3-6 April 2018 ; p. 790503.

156. Lindner, S.; Zhang, C.; Antolovic, I.M.; Pavia, J.M.; Wolf, M.; Charbon, E. Column-parallel dynamic TDC reallocation in SPAD sensor module fabricated in $180 \mathrm{~nm}$ CMOS for near infrared optical tomography. In Proceedings of the International Image Sensor Workshop, Hiroshima, Japan, 30 May-2 June 2017; pp. 86-89.

157. Kalyanov, A.; Jiang, J.; Lindner, S.; Ahnen, L.; di Costanzo, A.; Pavia, J.M.; Majos, S.S.; Wolf, M. Time domain near-infrared optical tomography with timeof-flight SPAD camera: The new generation. In Proceedings of the Biophotonics Congress: Biomedical Optics Congress, Hollywood, FL, USA, 3-6 April 2018; OF4D.5.

158. Ulku, A.C.; Bruschini, C.; Michalet, X.; Weiss, S.; Charbon, E. A $512 \times 512$ SPAD image sensor with built-in gating for phasor based real-time siFLIM. Proc. Int. Image Sens. Workshop 2017, 25, 234-237.

159. Ulku, A.C.; Bruschini, C.; Antolovic, I.M.; Kuo, Y.; Ankri, R.; Weiss , S.; Michalet , X.; Charbon, E. A 512 × 512 SPAD image sensor with integrated gating for widefield FLIM. IEEE J. Sel. Top. Quantum Electron. 2019, 25, 1-12. [CrossRef]

160. Ulku, A.C.; Bruschini, C.; Antolovic, I.M.; Weiss, S.; Michalet, X.; Charbon, E. Phasor-based widefield FLIM using a gated $512 \times 512$ single photon SPAD imager. Proc. SPIE 2019, 1088, 10882M.

161. Gyongy , I.; Calder, N.; Davies, A.; Dutton, N.A.W.; Duncan, R.R.; Rickman, C.; Dalgarno, P.; Henderson, R.K. A 256 × 256, 100 kfps, 61\% fill-factor SPAD image sensor for time-resolved microscopy applications. IEEE Trans. Electron Devices 2018, 65, 547-554. [CrossRef]

162. Hirose, Y.; Koyama, S.; Okino, T.; Inoue, A.; Saito, S.; Nose, Y.; Ishii, M.; Yamahira, S.; Kasuga, S.; Mori, M.; et al. A $400 \times 400-$ pixel $6 \mu \mathrm{m}$-pitch vertical avalanche photodiodes CMOS image sensor based on 150 ps-fast capacitive relaxation quenching in Geiger mode for synthesis of arbitrary gain images. In Proceedings of the IEEE International Conference on Solid-State Circuits Conference, San Francisco, CA, USA, 17-21 February 2019; pp. 104-105.

163. Henderson, R.K.; Johnston, N.; Hutchings, S.W.; Gyongy, I.; Al Abbas, T.; Dutton, N.; Tyler, M.; Chan, S.; Leach, J. A $256 \times 256$ $40 \mathrm{~nm} / 90 \mathrm{~nm}$ CMOS 3D-stacked $120 \mathrm{~dB}$ dynamic-range reconfigurable timeresolved SPAD imager. In Proceedings of the IEEE International Conference on Solid-State Circuits Conference, San Francisco, CA, USA, 7-21 February 2019; pp. $106-107$.

164. Morimoto, K.; Wu, M.L.; Ardelean, A.; Charbon, E. Superluminal Motion-Assisted Four-Dimensional Light-in-Flight Imaging. Phys. Rev. X 2021, 11, 011005.

165. Kekkonen, J.; Finnilä, M.A.J.; Heikkilä, J.; Anttonen V.; Nissinen, I. Chemical imaging of human teeth by a time-resolved Raman spectrometer based on a CMOS single-photon avalanche diode line sensor. Analyst 2019, 144, 6089-6097. [CrossRef]

166. Wahl, M.; Röhlicke, T.; Rahn, H.J.; Erdmann, R.; Kell, G.; Ahlrichs, A.; Kernbach, M.; Schell, A.W.; Benson, O. Integrated multichannel photon timing instrument with very short dead time high throughput. Rev. Sci. Instrum. 2013, 84, 043102. [CrossRef]

167. Rinnenthal, J.L.; Bornchen, C.; Radbruch, H.; Andresen, V.; Mossakowski, A.; Siffrin, V.; Seelemann, T.; Spiecker, H.; Moll, I.; Herz, J.; et al. Parallelized TCSPC for dynamic intravital fluorescence lifetime imaging: Quantifying neuronal dysfunction in neuroinflammation. PLoS ONE 2013, 8, e60100. [CrossRef]

168. Blacksberg, J.; Alerstam, E.; Cochrane, C.J.; Maruyama, Y.; Farmer, J.D. Miniature high-speed, low-pulse-energy ps Raman spectrometer for identification of minerals and organics in planetary science. Appl. Opt. 2020, 59, 433-444. [CrossRef]

169. Usai, A.; Finlayson, N.; Gregory, C.D.; Campbell, C.J.; Henderson, R.K. Separating fluorescence from Raman spectra using a CMOS SPAD TCSPC line sensor for biomedical applications. Proc. SPIE 2019, 10873, 108730R. 
170. Ferraro, J.R.; Nakamoto, K.; Brown, C.W. Introductory Raman Spectroscopy, 2nd ed.; Academic Press: Amsterdam, The Netherlands, 2003.

171. Hernandez, G. Fabry-Perot Interferometers; Cambridge University Press: Cambridge, UK, 1988.

172. Lee, J.Y.; Hahn, J.W.; Lee, H.-W. Spatiospectral transmission of a plane-mirror Fabry-Perot interferometer with non uniform finite-size diffraction beam illuminations. JOSA A 2002, 19, 973-984. [CrossRef]

173. Time-Correlated Single-Photon Counting Camera (Photon Force). Available online: https://www.photon-force.com/pf32-timeresolved-single-photon-counting-camera/ (accessed on 5 March 2021).

174. Single Photon Counting Camera (MPD). Available online: http://www.micro-photon-devices.com/MPD/media/UserManuals/ SPC3_usermanual.pdf (accessed on 5 March 2021).

175. Pellegrini, S.; Rae, B. Fully industrialised single photon avalanche diodes. Proc. SPIE 2017, 10212, $102120 \mathrm{D}$.

176. Sinfield, J.V.; Colic, O.; Fagerman, D.; Monwuba, C. A low cost time-resolved raman spectroscopic sensing system enabling fluorescence rejection. Appl. Spectrosc. 2010, 64, 201-210. [CrossRef] [PubMed]

177. Watanabe, J.; Kinoshita, S.; Kushida, T. Fluorescence rejection in Raman-spectroscopy by a gated single-photon counting method. Rev. Sci. Instrum. 1985, 56, 1195-1198. [CrossRef]

178. Ariese, F.; Meuzelaar, H.; Kerssens, M.M.; Buijsa, J.B.; Gooijera, C. ps Raman spectroscopy with a fast intensified CCD camera for depth analysis of diffusely scattering media Analyst. Def. Sci. J. 2009, 134, 1192-1197.

179. Andor Intensified CCDs for Time-Resolved Spectroscopy. Available online: https://andor.oxinst.com/assets/uploads / products/ andor/documents/andor-istar-ccd-spectroscopy-specifications.pdf (accessed on 8 March 2021).

180. Andor iStar sCMOS, Ultrafast Platform for Nanosecond Time-Resolved Imaging and Spectroscopy. Available online: https://andor.oxinst.com/assets/uploads/products/andor/documents/andor-istar-scmos-specifications.pdf (accessed on 8 March 2021).

181. Stanford ps High Speed ICCD Camera Family. Available online: https://stanfordcomputeroptics.com/products/ps-iccd.html\# technical-details (accessed on 8 March 2021)

182. Stanford Nanosecond High Speed ICCD Camera Family. Available online: https://stanfordcomputeroptics.com/products/ nanosecond-iccd.html\#technical-details (accessed on 8 March 2021).

183. Princeton PI-MAX 4. Available online: https://www.princetoninstruments.com/wp-content/uploads/2020/04/PIMAX4_1024i_ datasheet.pdf (accessed on 8 March 2021).

184. Gulati, K.K.; Gulia, S.; Gambhir, T.; Kumar, N.; Gambhir, V.; Reddy, M.N. Standoff Detection and Identification of Explosives and Hazardous Chemicals in Simulated Real Field Scenario using Time Gated Raman Spectroscopy. Def. Sci. J. 2019, 69, 342-347. [CrossRef]

185. Li, X.L.; Chen, Y.H.; Li, J.; Jiang, J.; Ni, Z.; Liu, Z.S. Time-resolved fluorescence spectroscopy of oil spill detected by ocean lidar. Proc. SPIE 2016, 10155, 101550Q.

186. Sawosz, P.; Wojtkiewicz, S.; Kacprzak, M.; Zieminska, E.; Morawiec, M.; Maniewski, R.; Liebert, A. Towards in-vivo assessment of fluorescence lifetime: Imaging using time-gated intensified CCD camera. Biocybern Biomed. Eng. 2018, 38, 966-974. [CrossRef]

187. Walczak, R.; Kubicki, W.; Dziuban, J. Low cost fluorescence detection using a CCD array and image processing for on-chip gel electrophoresis. Sens. Actuator B 2017, 240, 46-54. [CrossRef]

188. Dussault, D.; Hoess, P. Noise performance comparison of ICCD with CCD and EMCCD cameras. Proc. SPIE 2004, 5563, 195-204.

189. Angelini, F.; Di Frischia, S.; Chiuri, A.; Colao, F. Maximization of Raman signal in standoff detection under eye-safe conditions. Proc. SPIE 2019, 11166, 1116609.

190. Tkachenko, N. Optical Spectroscopy Methods and Instrumentations, 1st ed.; Elsevier Science: Amsterdam, The Netherlands, 2006.

191. Hamamatsu Streak Camera-Technical Information. Available online: https://www.hamamatsu.com/eu/en/product/ photometry-systems/streak-camera/related-documents/index.html (accessed on 5 March 2021).

192. Tahara T.; Hamaguchi, H.O. ps Raman Spectroscopy Using a Streak Camera. Appl. Spectrosc. 1993, 47, 391-398. [CrossRef]

193. Efremov, E.V.; Buijs, J.B.; Gooijer, C.; Ariese, F. Fluorescence rejection in resonance spectroscopy using a ps-gated intensified charge-coupled device camera. Appl. Spectrosc. 2007, 61, 571-578. [CrossRef]

194. Macleod, N.A.; Matousek, P. Deep Noninvasive Raman Spectroscopy of Turbid Media. Appl. Spectrosc. 2008, 62, 291-304. [CrossRef] [PubMed]

195. Hopkins, A.J.; Cooper, J.L.; Profeta, L.T.M.; Ford, A.R. Portable Deep-Ultraviolet (DUV) Raman for Standoff Detection. Appl. Spectrosc. 2016, 70, 861-873. [CrossRef] [PubMed]

196. Hain, R.; Kahler, C.J.; Tropea, C. Comparison of CCD, CMOS and intensified cameras. Exp. Fluids 2007, 42, 403-411. [CrossRef]

197. Genack, A.Z. Electro-optic phase-sensitive detection of optical-emission and scattering. Appl. Phys. Lett. 1985, 46, $341-343$. [CrossRef]

198. Demas, J.N.; Keller, R.A. Enhancement of luminescence and Raman-spectroscopy by phase-resolved background suppression. Anal. Chem. 1985, 57, 538-545. [CrossRef]

199. Wirth, M.J.; Chou, S.H. Comparison of time and frequency-domain methods for rejecting fluorescence from Raman-spectra. Anal. Chem. 1988, 60, 1882-1886. [CrossRef]

200. Canetta, E.; Mazilu, M.; DeLuca, A.C.; Carruthers, A.E.; Dholakia, K.; Neilson, S.; Sargeant, H.; Briscoe, T.; Herrington, C.S.; Riches, A.C. Modulated Raman spectroscopy for enhanced identification of bladder tumor cells in urine samples. J. Biomed. Opt. 2011, 16, 037002. [CrossRef] 
201. Praveen, B.B.; Steuwe, C.; Mazilu, M.; Dholakia, K.; Mahajan, S. Wavelength modulated surface enhanced (resonance) Raman scattering for background-free detection. Analyst 2013, 138, 2816-2820. [CrossRef] [PubMed]

202. Bell, S.E.J.; Bourguignon, E.S.O.; Dennis, A. Analysis of luminescent samples using subtracted shifted Raman spectroscopy. Analyst 1998, 123, 1729-1734. [CrossRef]

203. Osticioli, I.; Zoppi, A.; Castellucci, E.M. Shift-excitation Raman difference spectroscopy-difference deconvolution method for the luminescence background rejection from Raman spectra of solid samples. Appl. Spectrosc. 2007, 61, 839-844. [CrossRef] [PubMed]

204. Hasegawa, T.; Nishijo, J.; Umemura, J. Separation of Raman spectra from fluorescence emission background by principal component analysis. Chem. Phys. Lett. 2000, 317, 642-646. [CrossRef]

205. Zhao, J.; Lui, H.; McLean, D.I.; Zeng, H. Automated autofluorescence background subtraction algorithm for biomedical Raman spectroscopy. Appl. Spectrosc. 2007, 61, 1225-1232. [CrossRef]

206. Lieber, C.A.; Mahadevan-Jansen, A. Automated method for subtraction of fluorescence from biological Raman spectra. Appl. Spectrosc. 2003, 57, 1363-1367. [CrossRef]

207. Mosier-Boss, P.A.; Lieberman, S.H.; Newbery, R. Fluorescence rejection in Raman spectroscopy by shifted-spectra, edge-detection, and FFT filtering techniques. Appl. Spectrosc. 1995, 49, 630-638. [CrossRef]

208. Zhang, D.M.; Ben-Amotz, D. Enhanced chemical classification of Raman images in the presence of strong fluorescence interference. Appl. Spectrosc. 2000, 54, 1379-1383. [CrossRef]

209. Mazet, V.; Carteret, C.; Brie, D.; Idier, J.; Humbert, B. Background removal from spectra by designing and minimising a non-quadratic cost function. Chemom. Intell. Lab. Syst. 2005, 76, 121-133. [CrossRef]

210. Galloway, C.M.; LeRu, E.C.; Etchegoin, P.G. An iterative algorithm for background removal in spectroscopy by wavelet transforms. Appl. Spectrosc. 2009, 63, 1370-1376. [CrossRef] [PubMed]

211. Hu, Y.G.; Jiang, T.; Shen, A.G.; Li, W.; Wang, X.P.; Hu, J.M. A background elimination method based on wavelet transform for Raman spectra. Chemom. Intell. Lab. Syst. 2007, 85, 94-101. [CrossRef]

212. Leger, M.N.; Ryder, A.G. Comparison of derivative preprocessing and automated polynomial baseline correction method for classification and quantification of narcotics in solid mixtures. Appl. Spectrosc. 2006, 60, 182-193. [CrossRef] [PubMed]

213. Cao, A.; Pandya, A.K.; Serhatkulu, G.K.; Weber, R.E.; Dai, H.; Thakur, J.S.; Naik, V.M.; Naik, R.; Auner, G.W.; Rabah, R.; et al A robust method for automated background subtraction of tissue fluorescence. J. Raman Spectrosc. 2007, 38, 1199-1205. [CrossRef] 